\title{
Quantity, quality, equality: introducing a new measure of social welfare
}

\section{Karin Enflo ${ }^{1}$}

Received: 17 January 2019 / Accepted: 12 September 2020 / Published online: 16 April 2021

(c) The Author(s) 2021

\begin{abstract}
In this essay I propose a new measure of social welfare. It captures the intuitive idea that quantity, quality, and equality of individual welfare all matter for social welfare. More precisely, it satisfies six conditions: Equivalence, Dominance, Quality, Strict Monotonicity, Equality and Asymmetry. These state that (i) populations equivalent in individual welfare are equal in social welfare; (ii) a population that dominates another in individual welfare is better; (iii) a population that has a higher average welfare than another population is better, other things being equal; (iv) the addition of a well-faring individual makes a population better, whereas the addition of an ill-faring individual makes a population worse; (v) a population that has a higher degree of equality than another population is better, other things being equal; and (vi) individual illfare matters more for social welfare than individual welfare. By satisfying the six conditions, the measure improves on previously proposed measures, such as the utilitarian Total and Average measures, as well as different kinds of Prioritarian measures.
\end{abstract}

\section{Introduction}

Quantity, quality, and equality of individual welfare-these are the three factors most commonly held to affect the social welfare of a population. However, no extant measure of social welfare accommodates all three. The Total Measure accommodates only quantity. The Average Measure accommodates only quality. Prioritarian versions of these measures accommodate equality, but fail to accommodate either quantity or quality. Assuming that all three factors are important, a different kind of measure is thus called for.

In this essay I present a new measure (or strictly speaking a class of measures) that accommodates all three factors. The measure conjoins two separate measures of positive and negative social welfare. Each of these, as well as their aggregate, satisfies

Karin Enflo

karin.enflo@philosophy.su.se

1 Department of Philosophy, Stockholm University, SE 10691 Stockholm, Sweden 
at least five plausible conditions. In this respect the measure does better than the Total and Average measures, which satisfy only three of these conditions, and their prioritarian modifications, which satisfy only four. The five conditions are:

-An equivalence condition, according to which two populations that are equivalent in terms of individual welfare are ranked as equal in terms of social welfare.

-A dominance condition, according to which a population that dominates another in terms of individual welfare is ranked above the dominated population.

-A quality condition, according to which a population with higher average welfare is ranked above a population with lower average welfare, in comparisons between populations with the same total welfare and maximal degree of equality.

-A strict monotonicity condition, according to which the addition of an individual with positive welfare results in a higher ranked population, and the addition of an individual with negative welfare results in a lower ranked population.

-An equality condition, according to which a population with a higher degree of equality (in a specific sense of "equality") is ranked above a population with a lower degree of equality, in comparisons between populations with the same total and average welfare.

In addition to these five conditions, the conjoint measure also satisfies:

-An asymmetry condition, according to which negative individual welfare affects social welfare more than positive individual welfare, when their absolute values are equal.

These conditions are individually plausible and mutually consistent, but their joint satisfaction is nevertheless a challenge. The measure consequently has a somewhat complicated form. It will be presented later in this essay, the plan of which is as follows: in the next section I make some necessary assumptions regarding the measurability of individual and social welfare; in the third section I present the conditions concerning quantity, quality and equality; in the fourth section I introduce the measure and explain its design; in the fifth section I address some possible objections, and in the sixth section I make some concluding remarks. Proofs follow in the appendix.

\section{Preliminaries}

I will assume that the social welfare of a population depends on the individual welfare levels of its members. As this implies that a measure of social welfare must be a function of a measure of individual welfare, we should look at the measurability of individual welfare first.

Here I will assume that at any limited time interval, individuals fare either neutrally, or well or poorly to some degree. Individual welfare is thus a quantitative bipolar property with a neutral point. This is sufficient to make individual welfare measurable in theory and I will assume that such a measure exists. There is thus a function $v$ that assigns real numbers to all individuals, directly representing their levels of welfare, and indirectly representing relations between their levels of welfare. I will further assume that the measure is ratio scale, allowing representation of all kinds of quantitative 
relations between levels of welfare (ratio relations as well as difference and ordering relations). Positive welfare is represented by positive numbers, negative welfare by negative numbers, and neutral welfare by 0 . I assume that the relation does-better-than is asymmetric and transitive, while the relation does-equally-well-as is symmetric and transitive. I also assume interpersonal comparability.

The assumption that individual welfare is a bipolar property is consistent with most theories of individual welfare, as most theories distinguish between faring well and faring poorly. Some theories base these distinctions on the difference between goods and bads and then postulate that people fare well if the good in their lives outweighs the bad, and poorly if the bad in their lives outweighs the good. ${ }^{1}$ Other theories base these distinctions on the difference between faring better and faring worse and then postulate that people fare well if they fare better than some neutral level and poorly if they fare worse than the same neutral level. ${ }^{2}$

The assumption that individual welfare has a neutral point is also consistent with most theories of individual welfare. The neutral point represents the level of welfare of people who fare neither well nor poorly. A theory that is based on the distinction between goods and bads identifies the neutral point with the welfare level of people who either completely lack goods or bads in their lives, or have them to a degree where they balance each other out. ${ }^{3}$ A theory that is based on the distinction between faring better and faring worse need not identify a neutral point, but often does so in order to distinguish between faring well and faring poorly. ${ }^{4}$

I will further assume that, at some limited time interval, there is a lowest possible level of individual welfare that is still positive $\left(v_{p}\right.$ min $)$, and a highest possible level of individual welfare that is still negative $\left(v_{n} \max \right)$. In addition, there is a lowest as well as a highest possible level of individual welfare ( $v_{n}$ min and $v_{p}$ max). There is thus a lowest and a highest number that the measure of individual welfare assigns. Whatever these numbers are, the value assigned to $v_{p} \max$ should be at least double the value assigned to $v_{p}$ min, whereas the value assigned to $v_{n}$ min should be at least double the value assigned to $v_{n} \max$. (The technical reasons for these assumptions will be explained later.)

The assumption that individual welfare has bounds is consistent with most theories of individual welfare, as most theories postulate goods and bads that cannot be infinitely large or infinitely small. This is the case for all theories that postulate goods and bads that depend on psychological features, as such features naturally have bounds. There are limits to pleasures and pains, virtues and vices, needs and harms, wishes and fears, etc.

Moving on to social welfare, I will assume that it too is a quantitative bipolar property with a neutral point. This also makes social welfare measurable in theory, and I will assume that such a measure can be found. It would be a function $W$ assigning real numbers to populations, directly representing their levels of welfare, and indirectly representing relations between their levels of welfare. Positive welfare would

\footnotetext{
${ }^{1}$ See for example Brülde (2007: 2).

${ }^{2}$ See for example Broome (1999: 166-167).

${ }^{3}$ See Broome (1999: 170).

${ }^{4}$ See Broome (1999: 166).
} 
be represented by positive numbers, negative welfare by negative numbers, and neutral welfare by 0 . Again, the relation does-better-than would be asymmetric and transitive, whereas the relation does-equally-well-as would be symmetric and transitive.

Here I will assume that social welfare depends on individual welfare without qualifications, meaning that there are no levels of individual welfare too insignificant to contribute to social welfare. (Not everyone agrees with this, ${ }^{5}$ but the alternative view is problematic, both because there seems to be little reason for a certain level of welfare to contribute to social welfare when an adjacent level of welfare does not, and because there seems to be little reason for an individual welfare level to be too small to matter for social welfare when it is not too small to matter for the individual.)

I should add that I will use the term "welfare" for positive and negative welfare, when represented by positive and negative numbers. The term "illfare" will be used for negative welfare, when represented by positive numbers. This applies to both individual and social welfare. Also: I will often denote the social welfare of a population $A$ by $W(A)$ although the proper function form is rather $W(A, v)$.

\section{Conditions}

In this section I present the six conditions concerning how populations should be ranked in terms of social welfare. The conditions will be discussed relative to each relevant factor, beginning with quality. Underlying the conditions is an assumption that quantity, quality and equality all matter for social welfare-an assumption that I will not argue for in this essay.

\subsection{Conditions concerning quality}

The idea that quality matters for social welfare is hardly controversial. Surely, if the members of a population fare better, the population itself does better (everything else being equal). ${ }^{6}$ This idea can be understood in several different ways, however. Quality of individual welfare can be understood as either a vector property or a scalar property of populations. In the first case, quality is represented by a vector of numbers, showing the welfare of each individual. In the second case, quality is represented by a single number, which could be selected in different ways. Most commonly, average welfare is chosen. Here I will consider both kinds of quality, beginning with quality as a vector property.

It is well-known that vector properties are not straightforwardly comparable in the same way as scalar properties. If we regard quality of welfare as a vector property, there are only a few cases where we can confidently rank populations on its basis. One such case is the following: If two populations are equivalent in terms of individual

\footnotetext{
5 For example, Blackorby and Donaldson propose that there are insignificant levels above the neutral level (1984: 21).

6 This view has been held by Leibniz (1700/1768: 273), Hutcheson (1738: 181), Bentham (1823: 51), Wicksell (1910: 208), Broad (1930: 250), Gauthier (1963: 126), Narveson (1967: 219), Sikora (1975: 412), and Österberg (2006: 100), among others.
} 
welfare, then they should be ranked as equal in terms of social welfare. This idea can be more precisely presented as follows:

Equivalence Condition: For a measure of social welfare $W$ and for all possible populations $A$ and $B$ and their members $p_{i} \in A$ and $q_{i} \in B$, such that $|A|=|B|$, if there is a bijection from $A$ to $B$, such that each individual $p_{i} \in A$ could be paired with an individual $q_{i} \in B$ so that for each pair of individuals $\left(p_{i}, q_{i}\right)$ it is the case that $v\left(p_{i}\right)=v\left(q_{i}\right)$, then $A$ does equally well as $B$, and thus $W(A)=W(B){ }^{7}$

To illustrate: If $A$ and $B$ have three members each, and the welfare levels of their members are represented by the vectors $\mathbf{v}_{A}=(12,16,14)$ and $\mathbf{v}_{B}=(16,14,12)$, a measure of social welfare should rank $A$ and $B$ as equal in terms of social welfare.

The Equivalence Condition implies that it does not matter for social welfare how a set of welfare values is distributed among individuals. Thus the identities of the individuals do not matter, and neither do any of their properties (apart from their levels of welfare). This makes the condition consonant with the idea that each person's welfare matters equally to social welfare.

Another and almost as uncontroversial comparison is this: if one population strictly dominates another in terms of individual welfare, then the former should be ranked as better in terms of social welfare. This idea can be captured by the following condition:

Dominance Condition: For a measure of social welfare $W$ and for all possible populations $A$ and $B$ and their members $p_{i} \in A$ and $q_{i} \in B$, such that $|A|=|B|$, if there is a bijection from $A$ to $B$, such that each individual $p_{i} \in A$ could be paired with an individual $q_{i} \in B$ so that for each pair of individuals $\left(p_{i}, q_{i}\right)$ it is the case that $v\left(p_{i}\right) \geq v\left(q_{i}\right)$, and there is at least one pair of individuals $\left(p_{j}, q_{j}\right)$ for which it is the case that $v\left(p_{j}\right)>v\left(q_{j}\right)$, then $A$ does better than $B$, and thus $W(A)>W(B){ }^{8}$

To illustrate: If $A$ and $B$ have three members each, where $\mathbf{v}_{A}=(16,12,14)$ and $\mathbf{v}_{B}=(14,14,12)$, a measure of social welfare should $\operatorname{rank} A$ over $B$.

The Dominance Condition implies that quality is more important than equality for social welfare. That is, it is more important for social welfare that each individual fare as well as possible than that individuals fare equally well. Since even egalitarians agree with this, it is not very controversial. ${ }^{9}$

Having exhausted the (more or less) uncontroversial comparative possibilities for quality regarded as a vector property, I now move on to quality regarded as a scalar property, in the form of average individual welfare.

In this context, where I have assumed that three factors influence social welfare, it is least controversial to propose an everything-else-being-equal type of condition,

\footnotetext{
7 The Equivalence Condition is similar to Sen's Anonymity (1977: 1546), Dasgupta's Symmetry (1988: 108), Arrhenius's Neutrality (2000: 121), and Broome's Impartiality (2004: 135).

8 The Dominance Condition is similar to Sen's Strong Pareto (1977: 1546), Parfit's Same Number Quality Claim (1984: 360), and Broome's Principle of Personal Good (1991: 165). It implies the Pareto Condition, first proposed by Pareto (1909: 33).

9 At any rate, many philosophers hold that egalitarians should accept the Dominance Condition (or the Pareto Condition), for example Tungodden (2003: 9), Christiano and Braynen (2008: 392), Holtug (2010: 171), Hirose (2009: 306), and Broome (2015: 220). Only Nagel (1991: 107) and (perhaps) Temkin (1993: 140) disagree.
} 
where average welfare is varied and the other two factors are held fixed. This avoids weighing the importance of the three factors against one another and thus makes the condition hard to deny. More precisely, the condition can be formulated as follows:

Quality Condition: For a measure of social welfare $W$ and for all possible populations $A$ and $B$ and their members $p_{i} \in A$ and $q_{i} \in B$, such that $|A|=n$ and $|B|=m, \Sigma v\left(p_{i}\right)=\Sigma v\left(q_{i}\right)$, and the members of $A$ all have the same level of welfare, and the members of $B$ all have the same level of welfare, if $\Sigma v\left(p_{i}\right) / n>$ $\Sigma v\left(q_{i}\right) / m$, then $A$ does better than $B$, and thus $W(A)>W(B)$.

Assuming maximal equality, the Quality Condition implies that it is better to distribute a fixed positive amount of welfare within a smaller population, whereas it is better to distribute a fixed negative amount within a larger. To illustrate: If $A$ has two members and $B$ has three, where $\mathbf{v}_{A}=(12,12)$ and $\mathbf{v}_{B}=(8,8,8)$, a measure of social welfare should rank $A$ over $B$. If $C$ has three members and $D$ has two, where $\mathbf{v}_{C}=(-8,-8,-8)$ and $\mathbf{v}_{D}=(-12,-12)$, a measure of social welfare should rank $C$ over $D$.

The Quality Condition emphasizes the importance of the welfare levels of individuals rather than the number of individuals for the welfare of populations. One could oppose the condition in several ways. One possibility would be to flatly deny that quality in itself matters to social welfare, claiming that it matters only by contributing to quantity of welfare. In that case $\mathbf{v}_{A}=(12,12)$ and $\mathbf{v}_{B}=(8,8,8)$ would be equally good. This idea, however, goes against a main assumption of this essay, which is that welfare levels of separate individuals are important in themselves. Another possibility would be to claim that people contribute positively to social welfare by their very existence. In that case the contribution of additional people could trump a higher average welfare, making $\mathbf{v}_{B}=(8,8,8)$ a better population than $\mathbf{v}_{A}=(12,12)$. This claim, however, has problematic implications. It implies that (i) a population with people who fare neither well nor poorly would have positive social welfare; (ii) this welfare could improve indefinitely just by adding more people who fare neither well nor poorly; and thus (iii) for each population of people who all fare very well there could be a better (and larger) population of people who all fare neither well nor poorly. All these implications are implausible.

Yet another aspect of quality is its positive and negative sides. These could matter equally to social welfare, or one side could matter more. It seems to me that illfare matters more. ${ }^{10}$ A population $\mathbf{v}_{A}=(-5,-5)$ is plausibly bad to a higher degree than a population $\mathbf{v}_{B}=(5,5)$ is good. Likewise, a change from $(-5,5)$ to $(0,5)$ is plausibly better than a change from $(-5,5)$ to $(-5,10)$-and not just for egalitarian reasons. If I am correct in this, the following condition holds:

Asymmetry Condition: For a measure of social welfare $W$ and for all possible populations $A$ and $B$ if there is a bijection from $A$ to $B$, such that each individual $p_{i}$ $\in A$ could be paired with an individual $q_{i} \in B$ so that for each pair of individuals

\footnotetext{
10 Most famously, Popper (1945: 284) thought that illfare is more important than welfare. Others who have accepted the same idea include Walker (1974: 426), Österberg (1996: 100), Carlson (1998: 301) and Brown (2007: 349-350).
} 
$\left(p_{i}, q_{i}\right)$ it is the case that $\left|v\left(p_{i}\right)\right|=\left|v\left(q_{i}\right)\right|$ and also the case that $v\left(p_{i}\right)>v\left(q_{i}\right)$, then $|W(A)|<|W(B)| .^{11}$

To illustrate: If $A$ has the individual welfare vector $\mathbf{v}_{A}=(16,12,14)$ and $B$ has the individual welfare vector $\mathbf{v}_{B}=(-16,-12,-14)$ then $A$ is good to a lesser degree than $B$ is bad.

Note that the Asymmetry Condition does not specify the degree to which illfare matters more than welfare. It only excludes the possibilities that they matter to the exact same degree and that welfare matters more. It is thus consistent with the ideas that illfare matters just slightly more than welfare, that it matters much more, and that it matters to some degree in between. Moreover, it is also consistent with the ideas that illfare matters exclusively or lexically above welfare for social welfare, although these ideas are not plausible in themselves. ${ }^{12}$ The first idea has the absurd implication that, for example $\mathbf{v}_{A}=(1,1,1)$ is as good as $\mathbf{v}_{c}=(16,12,14)$, which goes against the Dominance Condition. The second idea has the almost equally absurd implication that, for example, $\mathbf{v}_{D}=(16,16,-2)$ is worse than $\mathbf{v}_{E}=(1,1,-1)$-an implication that perhaps only an adherent of Leximin could tolerate. ${ }^{13}$

\subsection{Conditions concerning quantity}

Quantity of welfare is usually regarded as relevant to social welfare as well. If there is more individual welfare in a population, the population itself does better (everything else being equal). ${ }^{14}$ Just like quality, quantity could be regarded as either a vector property or a scalar property of populations. Regarded as a vector property, it would also be represented by a vector of numbers, representing the welfare of each individual. Since this would collapse the distinction between quality and quantity, I will consider quantity only as a scalar property, that is, as represented by the total amount of individual welfare.

The way in which total welfare matters for social welfare is, again, least controversially captured by an everything-else-being-equal type of condition, similar to the Quality Condition. It can be formulated thus:

Quantity Condition: For a measure of social welfare $W$ and for all possible populations $A$ and $B$ and their members $p_{i} \in A$ and $q_{i} \in B$, such that $|A|=n$ and $|B|=m, \Sigma v\left(p_{i}\right) / n=\Sigma v\left(q_{i}\right) / m$, and the members of $A$ and $B$ all have the same level of welfare, if $\Sigma v\left(p_{i}\right)>\Sigma v\left(q_{i}\right)$, then $A$ does better than $B$, and thus $W(A)>W(B)$.

To illustrate: If $A$ has three members and $B$ has two, where $\mathbf{v}_{A}=(12,12,12)$ and $\mathbf{v}_{B}=(12,12)$, a measure of social welfare should rank $A$ over $B$. Also, if $C$ has two

11 The Asymmetry Condition is similar to Griffin's Weak Negative Doctrine (which he does not endorse, however). See Griffin (1979: 51).

12 Arguments against the view that illfare matters exclusively can be found in Smart (1958: 542), Smart (1961: 19-20), Acton (1963: 84) and Griffin (1979: 48).

13 Leximin was proposed by Sen (1970: 138).

14 This view has been held by Leibniz (1700/1768: 273), Hutcheson (1738: 181), Bentham (1823: 52), Sidgwick (1874: 386), Edgeworth (1877: 39), Smart (1961: 18), Sumner (1978: 97), and Broome (2004: 137), among others. 
members and $D$ has three, where $\mathbf{v}_{C}=(-12,-12)$ and $\mathbf{v}_{D}=(-12,-12,-12)$, a measure of social welfare should rank $C$ over $D$.

The Quantity Condition seems plausible but unnecessarily restrictive. It only concerns comparisons between populations that have the same average welfare and maximal degree of equality. For these cases, a population with a higher total sum of welfare is higher ranked. Intuitively, however, a population with a higher total sum should be higher ranked also in other comparisons. For example: adding welfare to a population (without making anyone worse off) should make the population better. This should hold regardless of whether welfare is added to existing individuals or is added by bringing well-faring individuals into existence. Similarly, adding illfare to a population (without making anyone better off) should make the population worse, regardless of whether illfare is added to existing individuals or is added by bringing illfaring individuals into existence. The first parts of these intuitions are captured by the Dominance Condition. The last parts can be summarized in the following condition:

Strict Monotonicity Condition: For a measure of social welfare $W$ and for any possible population $A$ and any individual $q \notin A$, if $v(q) \geq 1$, then $A \cup\{q\}$ does better than $A$, and thus $W(A \cup\{q\})>W(A)$, and if $v(q) \leq-1$, then $A \cup\{q\}$ does worse than $A$, and thus $W(A \cup\{q\})<W(A) .{ }^{15}$

To illustrate: if $A$ is a population and if $q_{i}$ and $q_{j}$ are individuals that may be brought into existence with $v\left(q_{i}\right)=12$ and $v\left(q_{j}\right)=-12$, then $A \cup\left\{q_{i}\right\}$ would fare better than $A$ while $A \cup\left\{q_{j}\right\}$ would fare worse than $A$.

The positive part of the condition is more controversial than the negative part. Most philosophers accept the negative part (except for adherents of the Average Measure, who accept neither), but some philosophers accept only the negative part and reject the positive. ${ }^{16}$ It is far from clear that such an asymmetry can be motivated, however. If one rejects the positive part of the condition, one must either deny that quantity of welfare matters for social welfare or propose that something else matters in such a way that the population $A \cup\{q\}$ is no better than $A$, even though $q$ fares well and no one else fares any worse. None of these options is appealing.

\subsection{Conditions concerning equality}

The third factor usually considered important for social welfare is equality of welfare. If the total amount of welfare is more equally distributed among the members of a population, the population itself does better. ${ }^{17}$ The importance of equality to social welfare is least controversially captured by an everything-else-being-equal type of condition, similar to the Quality and Quantity conditions, as follows:

\footnotetext{
15 The positive part of the condition is similar to Anglin's Extra Person Obligation (1977: 754), Sikora's Pareto Plus Principle (1978: 116), and Ng's Mere Addition Principle (1989: 237-238). The negative part of the condition is similar to Arrhenius's Negative Mere Addition Principle (2000: 69).

16 This kind of axiological asymmetry is accepted by Locke (1987: 156), and Broome (2004: 145), among others. It is denied by Rachels (1998: 104), Tännsjö (2002: 357), Holtug (2004: 139), and Huemer (2008: 923).

17 This view has been held by Sidgwick (1874: 417), Broad (1930: 251), Gauthier (1963: 126), and Narveson (1967: 211), among others.
} 
Equality Condition: For a measure of social welfare $W$ and for all possible populations $A$ and $B$ and their members $p_{i} \in A$ and $q_{i} \in B$, such that $|A|=|B|$ $=n$ and $\Sigma v\left(p_{i}\right)=\Sigma v\left(q_{i}\right)$, if the welfare of the members of $A$ is more equally distributed than the welfare of the members of $B$, then $A$ does better than $B$, and thus $W(A)>W(B)$.

Any egalitarian would accept this condition. It is not clear what it implies, however, since it is not clear how the phrase "more equally distributed than" should be interpreted. One possibility is to interpret the condition using one of the classic inequality measures. ${ }^{18}$ This seems unnecessarily restrictive, however. In this context any interpretation may be regarded as acceptable, as long as it is provided by a measure that satisfies certain conditions.

There is no need here to go through the lengthy process of formulating conditions for an equality measure, finding a measure that satisfies them, using the measure to interpret the Equality Condition, and finding a social welfare measure that satisfies it. Instead we could directly formulate conditions for a social welfare measure that could also be used for an equality measure. If a social welfare measure would satisfy these conditions, it could be used as an equality measure, and could thus be regarded as satisfying an acceptable version of the Equality Condition.

Because there are many ways of understanding inequality, only a few conditions would be uncontroversial: those that rank a more equally faring pair of individuals above a less equally fairing pair, and those that rank a maximally equal population above a less equal population (everything else being equal). These are the conditions that I will propose, beginning with the first kind:

Limited Equality Condition: For a measure of social welfare $W$ and for all possible populations $A$ and $B$ and their members $p_{i} \in A$ and $q_{i} \in B$, such that $|A|=|B|$ and $\Sigma v\left(p_{i}\right)=\Sigma v\left(q_{i}\right)$, if there is a bijection from $A$ to $B$, such that each individual $p_{i} \in A$ could be paired with an individual $q_{i} \in B$ so that for each pair of individuals $\left(p_{i}, q_{i}\right)$ it is the case that $v\left(p_{i}\right)=v\left(q_{i}\right)$, except for four individuals: $p_{1}, p_{2}, q_{1}, q_{2}$, such that $\left|v\left(p_{1}\right)-v\left(p_{2}\right)\right|<\left|v\left(q_{1}\right)-v\left(q_{2}\right)\right|$, then A does better than $B$, and thus $W(A)>W(B) .{ }^{19}$

To illustrate: If $A$ and $B$ have three members each, where $\mathbf{v}_{A}=(80,10,0)$ and $\mathbf{v}_{B}=(50,40,0)$, then $B$ does better than $A$ since there is a bijection from $A$ to $B$ such that for any two individuals, $p_{i} \in A$ and $q_{i} \in B$, it is the case that $v\left(p_{3}\right)=v\left(q_{3}\right)$ $(=0)$, except for four individuals where it is the case that $\left|v\left(p_{1}\right)-v\left(p_{2}\right)\right|>\mid v\left(q_{1}\right)-$ $v\left(q_{2}\right) \mid,(|80-10|>|50-40|)$.

Assuming (as I have) that the does-better-than relation is transitive, the Limited Equality Condition also implies a condition of the second kind:

\footnotetext{
18 See for example any of the measures by Pareto (1896), Gini (1912), Pietra (1915), and Theil (1967).

19 The Limited Equality Condition is similar to Hammond's Equity Axiom (1976: 795), although that condition applies to different outcomes for the same people. It is also similar to the famous Pigou-Dalton Condition, although that condition concerns transfers from better off people to worse off people (see Pigou (1912: 27) and Dalton (1920: 351)). Both conditions are satisfied by prioritarian measures that aggregate individual welfare by separable strictly concave functions.
} 
Maximal Equality Condition: For a measure of social welfare $W$ and for all possible populations $A$ and $B$ and their members $p_{i} \in A$ and $q_{i} \in B$, such that $|A|=|B|=n$ and $\Sigma v\left(p_{i}\right)=\Sigma v\left(q_{i}\right)$, if the welfare levels of the members of $A$ are all equal, and if the welfare levels of the members of $B$ are not all equal, then $A$ does better than $B$, and thus $W(A)>W(B) .^{20}$

To illustrate: If $A$ and $B$ have the same total sum of welfare of 90 , and three members each, where $\mathrm{v}_{A}=(30,30,30)$ and $\mathrm{v}_{B}=(60,20,10)$, then $A$ does better than $B$ because $A$ is maximally equally distributed and $B$ is not.

Both the Limited Equality Condition and the Maximal Equality Condition can be converted into conditions for an equality measure by substituting "equality" for "social welfare" and "is more equal than" for "does better than". If these conditions are sufficient to identify an equality measure and such a measure is used to interpret the Equality Condition, then any measure that satisfies the Limited Equality Condition also satisfies a version of the Equality Condition. In this sense, the Limited Equality Condition implies the Equality Condition as well.

\subsection{Summary of conditions}

A total of nine plausible and compatible conditions for a measure of social welfare have been discussed in this section: Equivalence, Dominance, Quality, Asymmetry, Quantity, Strict Monotonicity, Equality, Limited Equality, and Maximal Equality. Three conditions may be eliminated from further discussion: Quantity (since it follows from Strict Monotonicity, together with Equivalence), Maximal Equality (since it follows from Limited Equality, assuming transitivity), and Equality (since a measure that satisfies Limited Equality would also satisfy Equality, under the proposed interpretation). This leaves us with six conditions: Equivalence, Dominance, Quality, Asymmetry, Strict Monotonicity, and Limited Equality. Because these conditions apply to different comparative cases they cannot be further reduced. If quantity, quality, and equality matter for social welfare in the ways proposed, these are the conditions that a measure of social welfare should satisfy.

\section{The measure}

The six conditions are not sufficient to characterize a single measure and thus there are several measures that satisfy them. I will present one such measure in this section and briefly explain how it satisfies the conditions. First, however, I will comment on how some other measures fare with respect to the proposed conditions.

\subsection{Other measures}

The presentation of competing measures will be rather limited, as I shall consider only four:

\footnotetext{
20 The Maximal Equality Condition is similar to Blackorby et al.'s Weak Equality Preference (1998: 5) and Holtug's Egalitarian Relational Claim (2007: 128).
} 
- The Total Measure, which measures social welfare by the total sum of individual welfare. $^{21}$

- The Average Measure, which measures social welfare by the average level of individual welfare. $^{22}$

- The Prioritarian Total Measure, which measures social welfare by the total sum of individual welfare, weighted by a strictly concave function. ${ }^{23}$

- The Prioritarian Average Measure, which measures social welfare by the total sum of individual welfare, weighted by a strictly concave function, and divided by the cardinality of the population. ${ }^{24}$

Even though each of these measures satisfies some of the six conditions, none of them satisfies all six, as is shown in the following table:

\begin{tabular}{lllllll}
\hline & $\begin{array}{l}\text { Equivalence } \\
\text { condition }\end{array}$ & $\begin{array}{l}\text { Dominance } \\
\text { condition }\end{array}$ & $\begin{array}{l}\text { Quality } \\
\text { condition }\end{array}$ & $\begin{array}{l}\text { Asymmetry } \\
\text { condition }\end{array}$ & $\begin{array}{l}\text { Strict } \\
\text { Monotonic- } \\
\text { ity } \\
\text { condition }\end{array}$ & $\begin{array}{l}\text { Limited } \\
\text { Equality } \\
\text { condition }\end{array}$ \\
\hline $\begin{array}{c}\text { Total } \\
\text { measure }\end{array}$ & Yes & Yes & No & No & Yes & No \\
$\begin{array}{c}\text { Average } \\
\text { measure }\end{array}$ & Yes & Yes & Yes & No & No & No \\
$\begin{array}{c}\text { Prioritarian } \\
\text { total } \\
\text { measure }\end{array}$ & Yes & Yes & No & No & Yes & Yes \\
$\begin{array}{c}\text { Prioritarian } \\
\text { average } \\
\text { measure }\end{array}$ & Yes & Yes & Yes & No & No & Yes \\
\hline
\end{tabular}

Here I should add that many prioritarian measures apply only to positive welfare values and need to be adjusted to apply to negative values as well. If applied only to positive welfare values, a prioritarian measure would only satisfy the Equivalence Condition.

The measure that I will propose satisfies five of the proposed conditions partly in the same way as the above mentioned measures satisfy some of them. It satisfies the Equivalence, Dominance, and Strict Monotonicity conditions partly by aggregating all individual welfare values, like the Total Measure. It satisfies the Quality Condition partly by using a ratio between an aggregate function on individual welfare values and a function on the cardinality of the population, like the Average Measure. Finally, it satisfies the Limited Equality Condition partly by using a strictly concave function to aggregate individual welfare values, like the two Prioritarian measures.

\footnotetext{
21 The Total Measure has been endorsed by Sidgwick (1874: 386), Sikora (1978: 140), Sumner (1978: 97), and Huemer (2008: 928), among others.

22 The Average Measure has been endorsed by Harsanyi (1955: 316), and Pressman (2015: 389), among others.

23 The Prioritarian Total Measure has been endorsed by Sen (1973: 85), and Weirich (1983: 433), among others.

24 To my knowledge, the Prioritarian Average Measure has not been previously proposed in the literature, but is nevertheless useful to consider since it satisfies four of the six conditions.
} 
The main structural difference between the proposed measure and the other four is perhaps that the proposed measure uses two separate submeasures to aggregate positive and negative individual welfare, respectively. ${ }^{25}$ This allows the measure to satisfy the Asymmetry Condition, which the other four measures do not. However, all four measures could be adjusted to give negative welfare larger weight, so this is not an essential difference.

\subsection{The proposed measure}

Let us then consider the new measure. It has a rather complicated form and therefore I will present the measure in several steps, beginning with the overall measure and continuing with its positive and negative parts. First, let $W_{P}$ be a measure of positive social welfare, $W_{N}$ a measure of negative social welfare, $A$ a finite population, $p$ a member of $A, n$ the number of individuals in $A, v$ a measure of individual welfare, $v_{p} \max$ and $v_{n} \min$ the highest and lowest possible levels of individual welfare, $v_{p}$ min the lowest possible level of individual welfare that is still positive, and $v_{n}$ max the highest possible level of individual welfare that is still negative, $x$ the ratio between $v_{p} \max$ and $v_{p} \min$, and $y$ the ratio between $v_{n} \min$ and $v_{n} \max$. The overall social welfare measure $W$ is then defined as follows:

$$
\begin{aligned}
& W(A, v)=W_{P}(A, v)+W_{N}(A, v), \text { where: } \\
& W_{P}(A, v)=\frac{\sum_{i=1}^{n}\left(w\left(p_{i}\right)\right)^{r}}{n^{s}}, \text { where: } \\
& w\left(p_{i}\right)=\frac{v\left(p_{i}\right)}{v_{p} \min }, r=1-\frac{0.9}{2 x}, s=\frac{1}{2 x}, x=\frac{v_{p} \max }{v_{p} \min }, \text { and where: } v\left(p_{i}\right) \geq 0, n \geq 2, x \\
& \geq 2, \\
& \text { and } \\
& W_{N}(A, v)=\alpha \frac{-\sum_{i=1}^{n}\left(z\left(p_{i}\right)\right)^{t}}{n^{u}}, \text { where: } \\
& z\left(p_{i}\right)=\frac{v\left(p_{i}\right)}{v_{n} \max }, t=1+\frac{0.9}{2 y}, u=\frac{1}{2 y}, y=\frac{v_{n} \min }{v_{n} \max }, \text { where: } \alpha>1, v\left(p_{i}\right) \leq 0, n \geq 2, y \geq 2, \\
& v_{p} \min =\left|v_{n} \max \right| \text { and } v_{p} \max =\mid v_{n} \min .
\end{aligned}
$$

The first thing to note is that, strictly speaking, the measure is not a single measure but a class of measures, differing from one another regarding the values of $\alpha, v_{p}$ max, $v_{p} \min , v_{n} \max$, and $v_{n} \min$. This class can be reduced to one measure, when (if ever) these values become known. For now they are left unspecified.

The point of the weight $\alpha$, added only to the negative measure, and of the assumption that $\alpha>1$, is partly intuitive and partly technical. First, it accommodates the intuitive idea that negative welfare matters more for social welfare than positive welfare does and thus ensures that the measure satisfies the Asymmetry Condition. Secondly, it rewards equality for mixed positive and negative individual welfare values with (normalized) absolute values of 1 and thus ensures that the measure satisfies the Limited Equality Condition. It thus implies both that $\mathbf{v}_{A}=(1,1)$ is judged as less good than $\mathbf{v}_{B}=(-1,-1)$ is bad, and that $\mathbf{v}_{C}=(1,-1)$ is judged as worse than $\mathbf{v}_{D}=(0,0)$.

The assumption that $n \geq 2$ and the assumptions that $x \geq 2$ and $y \geq 2$ are needed for the measure to satisfy the Strict Monotonicity Condition. The measure thus only

\footnotetext{
25 Others who have proposed measures conjoining separate submeasures for positive and negative welfare include Carlson (1998), Österberg (2006), and Brown (2007).
} 
applies when a population has at least two members, and when the value of $v_{p}$ max is at least double the value of $v_{p} \min$, and the value of $v_{n} \min$ is at least double the value of $v_{n}$ max.

The assumptions that there are values $v_{p} \max , v_{p} \min , v_{n} \max$, and $v_{n} \min$ have several functions (which will be discussed in detail below). Most obviously, they are used to determine the concavity of the numerator of the positive social welfare measure, the convexity of the numerator of the negative social welfare measure, and the concavity of the denominator of both measures. If there were no values $v_{p} \max , v_{p} \min , v_{n} \max$, and $v_{n}$ min, the measure would not satisfy all of the conditions.

The specific assumptions that there are values $v_{p}$ min and $v_{n}$ max are needed also for a more general reason. Without these assumptions no continuous social welfare measure $W$ (depending on $v$ ) could satisfy the Strict Monotonicity, Quality and Limited Equality conditions together. This is easy to show. Let us assume that there is a population $A$ where everyone has positive welfare and let $x, y$, and $z$ denote any individuals such that $v(x)>0, v(y)=0, v(z)<0$ and $x, y, z \notin A$. According to the Strict Monotonicity Condition, $W(A \cup\{q\})>W(A)>W\left(A \cup\{z\}\right.$. If we do not assume any $v_{p}$ min and $v_{n}$ max for $v$, then, as $v$ approaches 0 , both $W(A \cup\{q\})$ and $W(A \cup\{z\})$ approach $W(A \cup\{y\})$ and thus we end up with $W(A)=W(A \cup\{y\})$. However, a social welfare measure that satisfies the Quality and Limited Equality conditions cannot equate $W(A)$ with $W(A \cup\{y\})$. This can be shown by an example.

Let us first assume that population $A$ has the welfare vector $\mathbf{v}_{A}=(12,12,12)$, and that population $B$ has the welfare vector $\mathbf{v}_{B}=(12,12,12,0)$. Let us then assume that there is a population $C$ with the same total welfare as $A$ and $B$, the same number of people as $B$, but maximally equal. $C$ thus has the welfare vector $\mathbf{v}_{C}=(9,9,9$, 9). According to the Equality Condition, $C$ is better than $B$, so $W(B)<W(C)$, and according to the Quality Condition, $A$ is better than $C$, so $W(C)<W(A)$. Thus, $W(A)>$ $W(C)>W(B)$. This, however, contradicts the result $W(A)=W(B)$, which we got when we assumed that there were no $v_{p} \min$ and $v_{n} \max$ for $v$. Thus, we must assume that there are $v_{p}$ min and $v_{n} \max$ for $v$, in order for any $W$ to satisfy the Strict Monotonicity, Quality and Limited Equality conditions, together.

The specific assumptions that there are values $v_{p}$ max and $v_{n}$ min are needed as well, although for a less general reason. Without these assumptions no continuous social welfare measure $W$ of the form $W(A)=f\left(v\left(p_{1}\right), v\left(p_{2}\right), \ldots, v\left(p_{n}\right)\right) / g(n)$, where $g(n)$ increases with $n$, could satisfy the Strict Monotonicity Condition. This will be shown for $v_{p} \max$ only (as the argument for $v_{n} \min$ is similar). To begin with, let us assume that the social welfare measure $W$ has the form $W(A)=f\left(v\left(p_{1}\right), v\left(p_{2}\right), \ldots, v\left(p_{n}\right)\right) / g(n)$, where $g(n)$ increases with $n$, and $f$ tends to infinity when any $v\left(p_{i}\right)$ tends to infinity. Let us next assume that there is a number $k>0$ such that $f\left(v\left(p_{1}\right), v\left(p_{2}\right), \ldots, v\left(p_{n}\right)\right.$, $v_{p}$ min $)<f\left(v\left(p_{1}\right), v\left(p_{2}\right), \ldots, v\left(p_{n}\right)\right)+k g(n+1)$. This means that there is a $k$ such that adding a member with individual welfare $v_{p} \min$ will not increase social welfare with more than $k$. Now, we can prove that without a $v_{p} \max$, the measure $W$ could not satisfy the Strict Monotonicity Condition.

To see this, let us consider a population $A$ with two members and a population $B$ with three members where $B=A \cup\{q\}$ and $v(q)=v_{p}$ min. The social welfare measure $W$ assigns $W(A)=f\left(v\left(p_{1}\right), v\left(p_{2}\right)\right) / g(2)$ and $W(B)=f\left(v\left(p_{1}\right), v\left(p_{2}\right), v_{p}\right.$ min $) / g(3)$. Since $g(n)$ increases with $n, g(3)>g(2)$. If $f\left(v\left(p_{1}\right), v\left(p_{2}\right)\right)=m$, then by the assump- 
tions made, $W(A)=m / g(2)$, and $f\left(v\left(p_{1}\right), v\left(p_{2}\right), v_{p}\right.$ min $)<m+k g(3)$, leading to $W(B)<(m+k g(3)) / g(3)=(m / g(2)) \times(g(2) / g(3))+k=W(A) \times(g(2) / g(3))+k$. Now, if $v\left(p_{1}\right)$ or $v\left(p_{2}\right)$ is sufficiently large, then $m>(g(2) k g(3)) /(g(3)-g(2))$, and thus $W(A)=m / g(2)>k g(3) /(g(3)-g(2))$. However, if $W(A)>k g(3) /(g(3)-g(2))$ then $W(A) \times(g(2) / g(3))+k<W(A)$ and since $W(B)<W(A) \times(g(2) / g(3))+k$, we end up with $W(B)<W(A)$. This contradicts the Strict Monotonicity Condition, which requires that $W(B)>W(A)$. Thus, we must assume that there are $v_{p} \max$ (and $v_{n} \min$ ) for $v$, in order for any $W$ (of the specified form) to satisfy the Strict Monotonicity Condition.

The assumptions that $v_{p} \min =\left|v_{n} \max \right|$ and that $v_{p}$ max $=\mid v_{n}$ min $\mid$ are made for convenience, since it gives the two submeasures the same denominator. Note that everyone in the population occurs in the denominator of both the positive and the negative part of the measure, whether they fare well, poorly, or neutrally.

Finally, the point of using $w\left(p_{i}\right)$ and $z\left(p_{i}\right)$, rather than just $v\left(p_{i}\right)$, is to normalize the measure, assigning a value of 1 to the minimal possible level of positive individual welfare, and a value of -1 to the maximal possible level of negative individual welfare. The number 1 thus functions as a unit of welfare.

There are more comments to be made regarding the form of the measure, but they relate separately to the positive and negative parts. Let us look at the positive part first.

\subsection{Measuring positive welfare}

The positive half of the proposed measure, $W_{P}$, which aggregates only positive individual welfare, has the following form:

$$
\begin{aligned}
& W_{P}(A, v)=\frac{\sum_{i=1}^{n}\left(w\left(p_{i}\right)\right)^{r}}{n^{s}}, \text { where: } \\
& w\left(p_{i}\right)=\frac{v\left(p_{i}\right)}{v_{p} \min }, r=1-\frac{0.9}{2 x}, s=\frac{1}{2 x}, x=\frac{v_{p} \max }{v_{p} \min }, \text { and where: } v\left(p_{i}\right) \geq 0, n \geq 2, x \\
& \geq 2 .
\end{aligned}
$$

Again, $A$ is a finite population, $p$ is a member of $A, n$ is the number of individuals in $A, v$ is a measure of individual welfare, and $v_{p}$ max and $v_{p}$ min are the highest and lowest possible levels of positive individual welfare, respectively.

Beginning with the numerator, it is an additively separable function on a vector of positive individual welfare values. This is the simplest kind of symmetric function, which ensures that the measure satisfies the Equivalence Condition. Because the function is strictly increasing, the measure satisfies the Dominance Condition, and because the function is strictly concave, the measure satisfies the Limited Equality Condition as well.

The denominator is a function of the cardinality of the population, which allows the measure to satisfy the Quality Condition. It also counters the strict subadditivity property of the concave function, which (implausibly) rewards the distribution of a fixed positive total sum of individual welfare values over more individual values.

For the measure to satisfy the Strict Monotonicity Condition, the denominator must grow at a slower rate than the numerator. This means that the total sum itself cannot be the numerator, and that cardinality itself cannot be the denominator. Instead, the numerator is raised to a power $(r)$ that approaches 1 , and the denominator is raised to 
a power $(s)$ that approaches 0 , as the ratio $v_{p} \max / v_{p} \min$ increases. As a consequence, the measure increasingly approximates the Total Measure.

The choices for $r$ and $s$ ensure that the measure satisfies various conditions. For the measure to satisfy the Limited Equality Condition, it is necessary to choose an $r<1$, ensuring strict concavity (where a smaller $r$ gives equality larger weight and a larger $r$ gives quality larger weight). For the measure to satisfy the Quality Condition, it is necessary to choose $r$ and $s$ such that $r+s>1$ (see proof in the Appendix). For the measure to satisfy the Strict Monotonicity Condition, $s$ must approach 0 as $x$ approaches infinity. It is therefore necessary for large $n$ that $s<2 / x^{r}$. This can be shown as follows: Let $A$ be a population with $n$ members, all of which have maximal individual welfare, $v_{p} \max =x$ (say). Let $B=A \cup\{q\}$ be a population with $n+1$ members, where all but $q$ have maximal individual welfare, and $q$ has minimal positive welfare, $v_{p} \min =1$. Then by the Strict Monotonicity Condition we have $W(A)=$ $n x^{r} / n^{s}<W(B)=\left(n x^{r}+1\right) /(n+1)^{s}$. We must then show that $n x^{r} / n^{s}<\left(n x^{r}+1\right) /(n+1)^{s}$. By multiplying both sides by $(n+1)^{s}$ we get: $(n+1)^{s} / n^{s} \times n x^{r}<n x^{r}+1 \ldots$ (I). An elementary inequality gives $(n+1)^{s} / n^{s}=(1+1 / n)^{s}>1+s / 2 n$. So for (I) to hold it is necessary that $(1+s / 2 n) \times n x^{r}<n x^{r}+1$, which is equivalent to $n x^{r}+s / 2 \times x^{r}$ $<n x^{r}+1$, which is equivalent to $s / 2 \times x^{r}<1$, which is $s<1 / 2 x^{r}$. These considerations make it convenient to choose $1 / 2 x$ for $s$, since it is simple and works for all $n$. Having made this choice for $s$, it is convenient to choose $1-0.9 / 2 x$ for $r$, since this is quite simple and close to as concave as this choice could be. (Unfortunately, the simpler and more concave choice $1-1 / 2 x$ does not work.)

\subsection{Measuring negative welfare}

At least from an aesthetic viewpoint, it would be nice if negative individual welfare could be aggregated in the same way as positive individual welfare. But for several reasons, it cannot. To see this, we may note that we could aggregate either negative welfare values or positive illfare values. Either choice would prevent us from using the same function as we used for positive welfare. The first choice would not give us a real valued social welfare measure. The second choice would not give us a measure that satisfies the Limited Equality Condition. This is because the measure of negative social welfare must be negative, and a negative of a concave function is a convex function, and convex functions do not reward equality.

In this context, where the positive illfare values of $z\left(p_{i}\right)$ are used rather than the negative welfare values of $v\left(p_{i}\right)$, individual illfare must be aggregated by a convex function. Here we can use a function that is similar to the concave function, although convex. If we do, the negative measure $W_{N}$ will look like this:

$$
\begin{aligned}
& W_{N}(A, v)=\alpha \frac{-\sum_{i=1}^{n}\left(z\left(p_{i}\right)\right)^{t}}{n^{u}}, \text { where: } \\
& z\left(p_{i}\right)=\frac{v\left(p_{i}\right)}{v_{n} \max }, t=1+\frac{0.9}{2 y}, u=\frac{1}{2 y}, y=\frac{v_{n} \min }{v_{n} \max }, \text { where: } \alpha>1, v\left(p_{i}\right) \leq 0, n \geq 2, y \geq 2 .
\end{aligned}
$$

Again, $A$ is a finite population, $p$ is a member of $A, n$ is the number of individuals in $A$, $v$ is a measure of individual welfare, and $v_{n} \max$ and $v_{n}$ min are the highest and lowest possible levels of negative individual welfare, respectively. 
Here the numerator is an additively separable function on a vector of positive individual illfare values. The measure satisfies the Equivalence Condition since the function is symmetric and the Dominance Condition since the function is strictly decreasing relative to illfare and thus strictly increasing relative to welfare. Because the numerator is the negative of a strictly convex function, it is strictly concave, and thus the measure satisfies the Limited Equality Condition as well.

The denominator of the negative measure is stipulated to be the same as for the positive measure, which facilitates adding the two. In this case too, the denominator allows the measure to satisfy the Quality Condition, although by rewarding a larger population rather than a smaller. The denominator also enhances the strict subadditivity property of the concave function, which (plausibly) rewards the distribution of a given negative total sum over more individual values.

Again, the denominator must grow at a slower rate than the numerator for the measure to satisfy the Strict Monotonicity Condition. In this case, the numerator is raised to a power $(t)$ that approaches 1 and the denominator is raised to a power $(u)$ that approaches 0 , as the ratio $v_{n} \min / v_{n} \max$ increases. Thus the negative measure increasingly approximates the Total Measure as well.

The choices for $t$ and $u$ ensure that the measure satisfies various conditions. For the measure to satisfy the Limited Equality Condition, it is necessary to choose a $t$ $>1$, ensuring strict convexity. For the measure to satisfy the Quality Condition, it is necessary to choose $t$ and $u$ such that $t+u>1$ (see proof in the Appendix). Here, $u$ has already been chosen to be the same as $s$ in the positive case, but $t$ cannot be chosen to be the same as $r$ (since $r<1$ ). However, it is possible to choose $t$ so that the negative measure is similar to the positive measure, that is: it is possible to choose $t$ so that $t=$ $1+0.9 / 2 y$, which is done here.

The weight $\alpha>1$ gives the negative measure larger weight than the positive measure. This weight is not specified (as it is not known). Technically, it assures that the measure satisfies the Asymmetry Condition and the Limited Equality Condition also for the special cases listed above. It also allows the negative measure to have much larger weight in comparison to the positive measure than what is possible by just aggregating illfare by a convex function and welfare by a concave function (as the convexity and concavity are restricted by other requirements).

\subsection{An example}

Before I go on to consider possible objections, let me briefly illustrate how the measure works. First, let us consider a very small population of four individuals, $A$, with individual welfare values given by the vector $\mathbf{v}_{A}=(10,10,0,-20)$. Let us assume that $v_{p} \max =20, v_{p} \min =0.1, v_{n} \max =-0.1, v_{n} \min =-20$, and that illfare has twice the weight of welfare so that $\alpha=2$.

We begin by normalizing the individual welfare values so that $\mathbf{v}_{A}=(10,10,0,-20)$ becomes $\mathbf{v}_{A}=(100,100,0,-200)$. Next, we calculate $x$ and $y$. Since $x=20 / 0.1$, and $y=-20 /-0.1$, both equal 200. This gives us $r=1-0.9 / 400=0.99775, s=1 / 400$ $=0.0025, t=1+0.9 / 400=1.00225, u=1 / 400=0.0025$. Inserting these values, 
the population's positive social welfare becomes $\left(100^{0.99775}+100^{0.99775}\right) / 4^{0.0025} \approx$ 197.3 , while its negative social welfare becomes $2\left(-\left(200^{1.00225} / 4^{0.0025}\right)\right) \approx-403.4$. Adding the two, we get: $W(A) \approx-206.1$. Thus, the population is doing poorly.

Next, let us compare the population $A$ with a few other small populations with the same total welfare: $B$ with welfare vector $\mathbf{v}_{B}=(20,0,-20), C$ with vector $\mathbf{v}_{C}=(10$, $10,0,-10,-10), D$ with vector $\mathbf{v}_{D}=(10,10,0,0,-20)$, and $E$ with vector $\mathbf{v}_{E}=(0$, $0,0,0)$. The above version of the measure assigns these populations the values $W(B)$ $\approx-206.6, W(C) \approx-205.4, W(D) \approx-206, W(E)=0$.

The measure thus ranks $E$ over $C$ over $D$ over $A$ over $B$. This illustrates the idea that quality and equality make a difference for social welfare (even when quantity is nil).

\section{Objections and replies}

By accommodating quantity, quality, and equality, in the ways specified by the six conditions, the proposed measure fares better than its main competitors. However, one may object that the measure does not accommodate the three factors in an altogether plausible way. The proposed measure is mostly responsive to quantity, less to quality, and even less to equality. This gives rise to some controversial rankings.

In this section I will consider two objections concerning how the measure weighs the three relevant properties. First, however, I will consider five other possible objections.

\subsection{Ad hoc-ness}

A first possible objection is that the measure seems ad hoc in that its design has no motivation other than accommodating the three factors in the ways specified by the six conditions. This critique presupposes that a measure should do more than assign numbers to populations to represent levels of social welfare. It should, perhaps, reflect intrinsic dependence relations between social welfare and other factors in its very form.

Now, the measure does reflect intrinsic dependence relations in its basic form: the conjoint form reflects that overall social welfare is a function both of social welfare and social illfare, the aggregation of all individual welfare values reflects that quantity matters for social welfare, and the use of a ratio between the aggregate individual welfare values and cardinality reflects that quality matters. The use of concave functions reflects the (conditional) importance of equality. In fact, the only details that do not reflect intrinsic dependence relations in this way are the values of $r, s, t$ and $u$. They are chosen only for the purpose of satisfying the six conditions. As regrettable as this may seem, there really are no better options. No simple and transparent measure will satisfy the six conditions. And abandoning any of the conditions because they can be jointly satisfied only by a measure that appears ad hoc seems even more ad hoc. 


\subsection{Arbitrariness}

A second possible objection is that the proposed measure is somewhat arbitrary in form. Although the general form of the measure is not arbitrary, the values of $r, s, t$ and $u$ need not be exactly as they are chosen here in order for the measure to satisfy the six conditions. For example: a variation, where $r$ is set to $1-0.99 / 2 x$, rather than $1-0.9 / 2 x$, or $t$ is set to $1+0.99 / 2 y$, rather than $1+0.9 / 2 y$, would also work (although less simple in notation).

Behind the arbitrariness worry lays the concern that if a proposed measure is in any way arbitrary we cannot be justified in thinking that the measure is correct. We can only be so justified if there is a reason for each detail of the proposed measure. In the ideal case, we should be able to list all conditions that are individually necessary and jointly sufficient to characterize the proposed measure and justify each one of them. In the present case, however, we might never be able to justify a condition that is able to fixate variables such as $r, s, t$ and $u$. Consequently, we might never be justified in thinking that the proposed measure is correct (above its many variations).

Considering that it is unlikely that we will ever be justified in selecting the proposed measure above its many variations, it might be preferable to include all possible variations of the measure in its very form. This is feasible, although it requires a more thorough mathematical investigation than I can undertake here.

\subsection{Insufficient specificity}

A third possible objection is that the proposed measure is insufficiently specified, since there are no exact values given for $\alpha, v_{p} \max , v_{p} \min , v_{n} \max$ and $v_{n} \min$. However, it would only be preferable to have these values specified if they were known. Since they are not known, it is rather a virtue of the proposed measure that it works with different sets of values.

\subsection{The larger weight of illfare}

A fourth possible objection is that the proposed measure is asymmetrical. It gives more weight to negative social welfare than to positive social welfare and it also gives more weight to individual illfare than to individual welfare.

However, either these asymmetries are correct. Then the new measure is also correct, at least in being asymmetrical. Alternatively, these asymmetries are not correct. Then there will still be infinitely many versions of the new measure that are almost correct, partly by aggregating individual illfare by just a slightly convex function, and partly by assigning a weight to negative social welfare that is just infinitesimally larger than 1. In either case, the measure does well. 


\subsection{The punishment of neutral additions}

A fifth possible objection is that the proposed measure ranks a population $A \cup\{q\}$ below $A$, when $q$ is faring neither well nor poorly (and the total welfare in $A$ is positive). Thus, additions that are neutral in terms of individual welfare are not treated as neutral in terms of social welfare. This might seem counterintuitive.

However, there are reasons not to yield to this objection. First, it does not seem clear (to me at least) that neutral additions are neutral for social welfare rather than bad (although it seems clear that they are not good). Secondly, and more importantly, the assumption that neutral additions are neutral for social welfare has unfortunate consequences. If we make this assumption, we cannot accept the Quality and the Limited Equality conditions together. This can easily be shown. First, we assume that $A$ is a population where all members fare well and equally well. Next, we assume that a neutrally faring individual is added to $A$ so that we get a population $B=A \cup\{q\}$. Last, we assume that $C$ has the same total welfare as $A$ and $B$, but that all members in $C$ fare equally well, unlike the members in B. According to the Quality Condition, $C$ is worse than $A$. According to the Limited Equality Condition, $C$ is better than $B$. The two conditions thus rank $A$ over $C$ over $B$. But since $B$ is worse than $A$, additions of neutrally faring individuals cannot be regarded as neutral-if we wish to accept the Quality and the Limited Equality conditions together.

\subsection{The weight of equality relative to quantity and quality}

A sixth possible objection is that the measure gives equality insufficient weight in comparison to quantity and quality. In fact, equality is given such a small weight that a more equal distribution rarely compensates for a lower total sum or a lower average welfare. This is due both to the use of concave functions, which give equality only a conditional effect on social welfare, and to the concave functions being only slightly concave. More generally, it is due to the measure being designed to satisfy the Strict Monotonicity Condition (which gives quantity larger weight than equality for some comparisons), the Dominance Condition (which gives quality larger weight than equality for other comparisons) and the Quality Condition (which combined with the other conditions limits the possibilities of the measure's design, see below).

Since we would not want to give up the Strict Monotonicity Condition, the Dominance Condition or the Quality Condition, equality must have lower weight than either quantity or quality in some cases. Even so, it might seem counterintuitive that it should have lower weight in cases not addressed by these conditions.

Unfortunately, this seems to be an example of how a social welfare measure cannot satisfy all our intuitions. I doubt that any continuous measure that satisfies the above conditions could give equality larger weight. As far as the proposed measure goes, the problem cannot be solved without getting into even worse problems. If we were to increase the concavity of the functions, equality would get larger weight, but the resulting measure would not satisfy the Strict Monotonicity Condition and the Quality Condition. If we would represent equality by a separate measure, and add or multiply that with a measure similar to the proposed measure, equality would also get larger 
weight, but this kind of measure would not satisfy the Strict Monotonicity Condition and the Dominance Condition. The first modification fails because the Quality Condition requires the variables $r$ and $s$ to be such that $r+s<1$ while the Strict Monotonicity Condition requires $s$ to be small, which means that they together require $r$ to be close to 1 , resulting in weak concavity. The second modification fails because the use of a separate measure for equality allows equality to trump additional welfare. Assuming that there are no better techniques that we could use, equality cannot get a larger weight.

\subsection{The weight of quality relative to quantity}

A final possible objection is that the proposed measure gives quality insufficient weight in comparison to quantity. Because of this, the measure does not avoid the repugnant conclusion. ${ }^{26}$ For any large possible population with very high levels of positive individual welfare there will always be a larger possible population with very low levels of positive individual welfare that is higher ranked. This seems repugnant not because it is in itself repugnant that people have low positive welfare, but because the population with better faring people intuitively should be higher ranked. ${ }^{27}$

Note that the proposed measure generally fares better than the Total Measure in terms of giving quality an appropriate weight. It would not rank a larger population as equal to a population with a higher average welfare, unless the larger population has a higher quantity of welfare. Neither would it rank a larger population as better than a population with a higher average welfare, unless the larger population is much larger than what the Total Measure requires. Unfortunately, this is insufficient to prevent the repugnant conclusion. Quantity of welfare has too large an impact on the measure's rankings for that.

There are several possible solutions to this problem. One option is to deny that the repugnant conclusion is indeed repugnant (which many philosophers have done, for different reasons). ${ }^{28}$ Another option is to grant that the repugnant conclusion is repugnant, although not sufficiently repugnant to warrant its denial. ${ }^{29} \mathrm{~A}$ third option is to modify the proposed measure so that the repugnant conclusion does not follow. Let us look at these three responses in turn.

One response is thus to deny that the conclusion is repugnant. People who disagree have made some kind of error. Perhaps they find it in itself repugnant that people have low positive welfare (since they fail to imagine what it would be like). ${ }^{30}$ Or perhaps

\footnotetext{
26 The problem was named by Parfit (1984: 388), although similar problems, with similar names, were brought up previously by Sidgwick (1874: 386), McTaggart (1927: 453), Broad (1930: 249-250), and Rawls (1971: 162-163). Similar problems concerning illfare have subsequently been brought up by Blackorby et al. (1998: 11), Tännsjö (2002: 340), and Mulgan (2002: 362).

27 See McMahan (1981: 119), Dasgupta (1988: 117), and Broome (2004: 212).

28 See Sikora (1978), Dasgupta (1988), Ng (1989), Hare (1993), Ryberg (1996), Tännsjö (2002), and Huemer (2008).

29 See Anglin (1977).

30 See Sikora (1978: 117), McMahan (1981: 119), Mackie (1985: 246), Dasgupta (1988: 117), Ryberg (1996: 143-144), Tännsjö (2002: 345), and Broome (2004: 212).
} 
they have difficulties evaluating large populations of people (since they fail to imagine large quantities of welfare). ${ }^{31}$ Whatever the reason, their intuitions are off.

A second response is to grant that the conclusion is repugnant, but argue that it should be accepted nonetheless. One could argue that a conclusion that follows from a set of reasonable assumptions should be reasonable as well. ${ }^{32}$ As the repugnant conclusion follows from a set of reasonable assumptions (including the idea that a population improves by adding welfare and the idea that welfare never diminishes in value) it should be okay.

A third response is to modify the proposed measure so that the conclusion does not follow. This choice may be more problematic than the repugnant conclusion itself, however. Let me give some examples of such possible modifications.

One way to avoid the repugnant conclusion would be to raise the numerators of the two submeasures to a power closer to 1 , rather than 0 . The resulting measure would be more similar to the Average Measure than the Total Measure and would avoid the conclusion in a similar way. Unfortunately, it would not satisfy the Strict Monotonicity Condition. Another way to avoid the repugnant conclusion would be to reduce the contributive value of individual welfare to social welfare so that each additional individual contributes less individual welfare. ${ }^{33}$ The values of this modified measure would approach some constant rather than infinity, and thus avoid the conclusion. Unfortunately, this measure would not satisfy the Equivalence Condition.

There are other solutions proposed in the literature as well: the idea that quantity has a maximal contributive value, or that quality has a low maximal value, or that low positive values do not contribute to social welfare at all. ${ }^{34}$ The first solution is inconsistent with the Strict Monotonicity Condition, the second solution does not solve the problem for all comparisons, and the third solution is inconsistent with both the Dominance Condition and the Strict Monotonicity Condition and does not solve the problem for all comparisons either.

Other types of solutions that appeal to for example value pluralism, value incommensurability, value indeterminateness, lexical values, or intransitivity of the betterness relation, are incompatible with the assumptions used in this essay, and so will not be discussed here. ${ }^{35}$

Thus: If we want a measure that satisfies the Equivalence and Strict Monotonicity conditions, and is consistent with the assumptions used in this essay, we most likely have to accept the repugnant conclusion.

\footnotetext{
31 See Anglin (1977: 748), Ng (1989: 242), Broome (2004: 212), and Huemer (2008: 910).

32 See Anglin (1977: 751), Ng (1989: 242), and Huemer (2008: 923).

33 See Parfit (1984: 402) and Cowen (1996: 762-763).

34 For the first solution, see Parfit (1984: 402) and Cowen (2004: 85); for the second, see Mackie (1985: 246); and for the third, see Parfit (1984: 412) and Blackorby and Donaldson (1984: 21).

35 For the first solution, see Parfit (1986: 163) and Cowen (1996: 758); for the second, see Parfit (1982: 164), Österberg (1996: 101), and Rabinowicz, (2009: 391); for the third, see Broome (2004: 172-173); for the fourth, see Mill (1867: 12), Parfit (1984: 414), Mackie (1985: 246), Griffin (1986: 85-86), Rachels (2001: 217), and Mulgan (2006: 64-81); and for the fifth, see Rachels (2001: 232) and Persson (2004: 198).
} 


\section{Concluding remarks}

So where do these objections leave us? The first objection is based on the dubious idea that a social welfare measure must be intuitively transparent. The following two objections could be met by gaining additional knowledge. The next two objections point to problems that might rather be virtues. Only the last two objections are really troubling. If they cannot be met, there may be only one way of dealing with them: by redesigning the measure in such a way that it no longer satisfies the six conditions.

However, if quantity, quality, and quality matter for social welfare (and nothing else matters), it seems difficult to abandon any of the six conditions. Equivalence seems indisputable. The order in which individual welfare values occur cannot matter for social welfare. The Quantity, Quality, and Equality conditions are on a par, and equally difficult to deny. Surely: if two factors are held fixed, the third will be decisive. The Dominance Condition does put quality above equality, but that everyone fare as well as possible seems more important than that everyone fare equally well. Asymmetry seems intuitive and abandoning it would not solve any major problems. This leaves us with Strict Monotonicity: perhaps it could go?

If Strict Monotonicity is abandoned we might at least avoid the repugnant conclusion. But it is far from clear that we should make this choice. It seems certain that bringing ill-faring persons into existence makes a population worse, and almost as certain that bringing well-faring persons into existence makes a population better. That a large population of slightly well-faring people cannot be better than a smaller population of very well-faring people seems rather less certain.

I am not going to insist on this point here, however. The problem discussed is just an example of a more general problem in population ethics: that we cannot accommodate all of our intuitions concerning social welfare, and thus have to make a choice. ${ }^{36}$ As difficult as such a choice may be, it need not be as difficult as that between quantity, quality, and equality of welfare. The measure proposed in this essay accommodates all three.

Acknowledgements This paper grew out of conversations with Per Enflo and Victor Moberger, to whom I am most grateful. Earlier versions were presented at the Higher Seminar in Practical Philosophy at Stockholm University in the fall of 2016, and at the Swedish Congress of Philosophy at Uppsala University in the summer of 2017. I am grateful to the participants for their comments, especially to Erik Angner and Daniel Ramöller. I am also grateful to several anonymous referees.

Funding Open access funding provided by Stockholm University.

Open Access This article is licensed under a Creative Commons Attribution 4.0 International License, which permits use, sharing, adaptation, distribution and reproduction in any medium or format, as long as you give appropriate credit to the original author(s) and the source, provide a link to the Creative Commons licence, and indicate if changes were made. The images or other third party material in this article are included in the article's Creative Commons licence, unless indicated otherwise in a credit line to the material. If material is not included in the article's Creative Commons licence and your intended use is not permitted by statutory regulation or exceeds the permitted use, you will need to obtain permission directly from the copyright holder. To view a copy of this licence, visit http://creativecommons.org/licenses/by/4.0/.

36 See Cowen (1996), Carlson (1998), and Arrhenius (2000). 


\section{Appendix}

\section{Proofs for the positive social welfare measure}

Let $A$ be a finite population, $p$ a member of $A, n$ the number of individuals in $A, v$ a measure of individual welfare, and $v_{p} \max$ and $v_{p}$ min the highest and lowest possible levels of positive individual welfare, respectively. Then we have:

The positive social welfare measure $W_{P}$

$$
\begin{aligned}
& W_{P}(A, v)=\frac{\sum_{i=1}^{n}\left(w\left(p_{i}\right)\right)^{r}}{n^{s}}, \text { where: } \\
& w\left(p_{i}\right)=\frac{v\left(p_{i}\right)}{v_{p} \min }, r=1-\frac{0.9}{2 x}, \mathrm{~s}=\frac{1}{2 x}, x=\frac{v_{p} \max }{v_{p} \min }, \text { and where: } v\left(p_{i}\right) \geq 0, n \geq 2, x \geq 2 .
\end{aligned}
$$

I will now show that $W_{P}$ satisfies the five conditions.

\section{Satisfaction of the equivalence condition}

Equivalence Condition: For a measure of social welfare $W$ and for all possible populations $A$ and $B$ and their members $p_{i} \in A$ and $q_{i} \in B$, such that $|A|=|B|$, if there is a bijection from $A$ to $B$, such that each individual $p_{i} \in A$ could be paired with an individual $q_{i} \in B$ so that for each pair of individuals $\left(p_{i}, q_{i}\right)$ it is the case that $v\left(p_{i}\right)=v\left(q_{i}\right)$, then $A$ does equally well as $B$, and thus $W(A)=W(B)$.

Proof The positive social welfare measure satisfies the Equivalence Condition since it is a symmetric function.

\section{Satisfaction of the dominance condition}

Dominance Condition: For a measure of social welfare $W$ and for all possible populations $A$ and $B$ and their members $p_{i} \in A$ and $q_{i} \in B$, such that $|A|=$ $|B|$, if there is a bijection from $A$ to $B$, such that each individual $p_{i} \in A$ could be paired with an individual $q_{i} \in B$ so that for each pair of individuals $\left(p_{i}, q_{i}\right)$ it is the case that $v\left(p_{i}\right) \geq v\left(q_{i}\right)$, and there is at least one pair of individuals $\left(p_{j}, q_{j}\right)$ for which it is the case that $v\left(p_{j}\right)>v\left(q_{j}\right)$, then $A$ does better than $B$, and thus $W(A)$ $>W(B)$.

Proof It is trivial that the positive social welfare measure satisfies the Dominance Condition, since $\sum_{i=1}^{n} w\left(p_{i}\right)^{r}$ is a strictly increasing function of $w\left(p_{i}\right)$ for every $i$ (and $w\left(p_{i}\right)=v\left(p_{i}\right) / v_{p}$ min $)$.

\section{Satisfaction of the quality condition}

Quality Condition: For a measure of social welfare $W$ and for all possible populations $A$ and $B$ and their members $p_{i} \in A$ and $q_{i} \in B$, such that $|A|=n$ and $|B|=m, \Sigma v\left(p_{i}\right)=\Sigma v\left(q_{i}\right)$, and the members of $A$ all have the same level of welfare, and the members of $B$ all have the same level of welfare, if $\Sigma v\left(p_{i}\right) / n>\Sigma v\left(q_{i}\right) / m$, then $A$ does better than $B$, and thus $W(A)>$ $W(B)$. 
Proof Let us use $w\left(p_{i}\right)=v\left(p_{i}\right) / v_{p}$ min, rather than $v\left(p_{i}\right)$. In this situation, $w\left(p_{i}\right) \geq 0$ and $w\left(q_{i}\right) \geq 0$, and all $w\left(p_{i}\right)$ and all $w\left(q_{i}\right)$ are equal. Since $\Sigma w\left(p_{i}\right) / n>\Sigma w\left(q_{i}\right) / m$, it is the case that $|A|<|B|$. It is sufficient to prove that the condition holds for $m=n+1$.

Let us then assume that $w\left(p_{i}\right)=k$, where $1 \leq i \leq n$, and that $w\left(q_{j}\right)=k \frac{n}{n+1}$, where $1 \leq j \leq n+1$. This gives:

$$
\sum_{i=1}^{n} w\left(p_{i}\right)=\sum_{j=1}^{n+1} w\left(q_{j}\right)
$$

We have to prove that:

$$
\frac{\sum_{i=1}^{n}\left(w\left(p_{i}\right)\right)^{r}}{n^{s}}>\frac{\sum_{j=1}^{n+1}\left(w\left(q_{j}\right)\right)^{r}}{(n+1)^{s}}
$$

This is the same as:

$$
\frac{n k^{r}}{n^{s}}>\frac{(n+1)\left(k \frac{n}{n+1}\right)^{r}}{(n+1)^{s}}
$$

To prove (2), we divide both sides by the left hand side. This gives that we must prove that:

$$
1>\frac{(n+1)\left(k \frac{n}{n+1}\right)^{r}}{(n+1)^{s}} \frac{n^{s}}{n k^{r}} .
$$

Since

$$
\frac{(n+1)\left(k \frac{n}{n+1}\right)^{r}}{(n+1)^{s}} \frac{n^{s}}{n k^{r}}=(n+1)^{1-r-s} n^{r+s-1}=\left(1+\frac{1}{n}\right)^{1-r-s}
$$

we have:

$$
1>\left(1+\frac{1}{n}\right)^{1-r-s}
$$

Since

$$
1-r-s=1-\left(1-\frac{0.9}{2 x}\right)-\left(\frac{1}{2 x}\right)=-\frac{0.1}{2 x}
$$

we have:

$$
\left(1+\frac{1}{n}\right)^{1-r-s}=\left(1+\frac{1}{n}\right)^{\frac{-0.1}{2 x}}<1
$$

and so (3) and with that (1) is proved. 


\section{Satisfaction of the strict monotonicity condition}

Strict Monotonicity Condition (positive part): For a measure of social welfare $W$ and for any possible population $A$ and any individual $q \notin A$, if $v(q) \geq 1$ then $A \cup\{q\}$ does better than $A$ and thus $W(A \cup\{q\})>W(A)$.

Proof Let us again use $w\left(p_{i}\right)=v\left(p_{i}\right) / v_{p}$ min instead of $v\left(p_{i}\right)$. To prove that the positive social welfare measure satisfies the Strict Monotonicity Condition, we need to prove:

$$
\frac{\sum_{i=1}^{n}\left(w\left(p_{i}\right)\right)^{r}}{n^{s}}<\frac{\sum_{i=1}^{n}\left(w\left(p_{i}\right)\right)^{r}+(w(q))^{r}}{(n+1)^{s}}
$$

Since $r>0$, it suffices to prove this for the case where $v(q)=v_{p} \min$, which means that $w(q)=1$. By dividing both sides in (4) by the left hand side, we see that to prove (4) it suffices to prove that:

$$
1<\frac{\sum_{i=1}^{n}\left(w\left(p_{i}\right)\right)^{r}+1}{(n+1)^{s}} \frac{n^{s}}{\sum_{i=1}^{n}\left(w\left(p_{i}\right)\right)^{r}} .
$$

This is the same as:

$$
1<\left(1+\frac{1}{n}\right)^{-s}\left(1+\frac{1}{\sum_{i=1}^{n}\left(w\left(p_{i}\right)\right)^{r}}\right)
$$

To prove (4) it suffices to prove (5), assuming that all $w\left(p_{i}\right)=w_{p} \max =x$, and that $1 \leq i \leq n$, that is, to prove:

$$
1<\left(1+\frac{1}{n}\right)^{-s}\left(1+\frac{1}{n}\left(\frac{1}{x}\right)^{r}\right), \text { where } x=\frac{v_{p} \max }{v_{p} \min }
$$

Since $s=\frac{1}{2 x}$ and $r=1-\frac{0.9}{2 x}$ this means that (6) becomes:

$$
1<\left(1+\frac{1}{n}\right)^{-\frac{1}{2 x}}\left(1+\frac{1}{n}\left(\frac{1}{x}\right)^{1-\frac{0.9}{2 x}}\right)
$$

We have to prove that (7) holds for all $x \geq 2, n \geq 2$. For this we use the elementary inequality:

$$
\left(1+\frac{1}{n}\right)^{-\frac{1}{2 x}}>1-\frac{1}{2 n x}
$$

By putting (8) into (7), we see that it suffices to prove:

$$
1<\left(1-\frac{1}{2 n x}\right)\left(1+\frac{1}{n x}\left(\frac{1}{x}\right)^{\frac{-0.9}{2 x}}\right)
$$


Putting $\left(\frac{1}{x}\right)^{\frac{-0.9}{2 x}}=x^{\frac{0.9}{2 x}}$ and doing the multiplication in the right hand side of (9) we get that (9) is equivalent to:

$$
1<1+\frac{1}{n x}\left(x^{\frac{0,9}{2 x}}-\frac{1}{2}\right)-\frac{1}{2 n^{2} x^{2}} x^{\frac{0.9}{2 x}}
$$

We then have:

$$
1.15<x^{\frac{0.9}{2 x}}<1.25, \text { for } 2 \leq x \leq 4, \text { and } x^{\frac{0.9}{2 x}}<1.25, \text { for } x \geq 4
$$

To see this we observe that $e^{\frac{0.9 \ln x}{2 x}}=x^{\frac{0.9}{2 x}}$ attains its maximum for $x=e$, with $1.15<$ $e^{\frac{0.9}{2 e}}<1.25$, and for $x=2$ and $x=4$ we have $1.15<2^{\frac{0.9}{4}}=4^{\frac{0.9}{8}}$.

To prove (9) for the case $2 \leq x \leq 4$, we see that by (10) and (11), the right hand side of (9) is larger than:

$$
1+\frac{1}{n x}(1.15-0.5)-1.25 \frac{1}{2 n^{2} x^{2}}=1+\frac{1}{n x}\left(0.65-1.25 \frac{1}{2 n x}\right) .
$$

We also see that:

$1+\frac{1}{n x}\left(0.65-1.25 \frac{1}{2 n x}\right) \geq 1+\frac{1}{n x}\left(0.65-1.25 \frac{1}{8}\right)>1$, which proves (9) for $2 \leq x$ $\leq 4$.

To prove (9) for $x \geq 4$, we observe that:

$$
x^{\frac{0.9}{2 x}}=e^{\frac{0.9 \ln x}{2 x}}>1+\frac{0.9 \ln x}{2 x} \text { for } x \geq 4, n \geq 2
$$

By (11) we have:

$$
x^{\frac{0.9}{2 x}}<1.25, \text { for } x \geq 4
$$

Using (10), (12) and (13) we see that the right hand side of (9) is larger than:

$$
1+\frac{1}{n x}\left(0.5+\frac{0.9 \ln x}{2 x}\right)-\frac{1.25}{2 n^{2} x^{2}}=1+\frac{1}{n x^{2}}\left(0.5 x+\frac{0.9 \ln x}{2}-\frac{1.25}{2 n}\right)>1 \text {. }
$$

So by this we see that (9) holds for all $x \geq 2, n \geq 2$, and thus it is proved that the positive social welfare measure satisfies the Strict Monotonicity Condition.

\section{Satisfaction of the limited equality condition}

Limited Equality Condition: For a measure of social welfare $W$ and for all possible populations $A$ and $B$ and their members $p_{i} \in A$ and $q_{i} \in B$, such that $|A|=|B|$ and $\Sigma v\left(p_{i}\right)=\Sigma v\left(q_{i}\right)$, if there is a bijection from $A$ to $B$, such that each individual $p_{i} \in A$ could be paired with an individual $q_{i} \in B$ so that for each pair of individuals $\left(p_{i}, q_{i}\right)$ it is the case that $v\left(p_{i}\right)=v\left(q_{i}\right)$, except for four individuals: $p_{1}, p_{2}, q_{1}, q_{2}$, such that $\left|v\left(p_{1}\right)-v\left(p_{2}\right)\right|<\left|v\left(q_{1}\right)-v\left(q_{2}\right)\right|$, then $A$ does better than $B$, and thus $W(A)>W(B)$. 
Proof Since the number of options is fixed, we only need to show that a separable strictly concave measure of the form:

$$
W(A)=\sum_{i=1}^{n} w\left(p_{i}\right)^{r}, \text { where } 3 / 4<r<1,
$$

satisfies the Limited Equality Condition (as noted: $A$ is a finite population, $p$ is a member of $A, w$ is a measure of welfare, and $n$ is the number of persons in $A$ ). Again, we are using $w\left(p_{i}\right)=v\left(p_{i}\right) / v_{p}$ min rather than $v\left(p_{i}\right)$.

Because $3 / 4<r<1$, the function $w\left(p_{i}\right)^{r}$ is strictly concave. Let us put $f=w\left(p_{i}\right)^{r}$. By hypothesis, $w\left(p_{1}\right)+w\left(p_{2}\right)=w\left(q_{1}\right)+w\left(q_{2}\right)$ and $\left|w\left(p_{1}\right)-w\left(p_{2}\right)\right|<\left|w\left(q_{1}\right)-w\left(q_{2}\right)\right|$, thus $w\left(p_{1}\right)-w\left(q_{1}\right)=w\left(q_{2}\right)-w\left(p_{2}\right)$. Suppose that $w\left(p_{1}\right)<w\left(p_{2}\right)$ and that $w\left(q_{1}\right)<$ $w\left(q_{2}\right)$. In this case, since $w\left(p_{i}\right)+w\left(p_{2}\right)=w\left(q_{1}\right)+w\left(q_{2}\right)$, we have $w\left(q_{1}\right)<w\left(p_{1}\right)<$ $w\left(p_{2}\right)<w\left(q_{2}\right)$.

We then apply the Mean Value Theorem. Assume that the function $f$ is continuous on the closed interval $\left[w\left(q_{1}\right), w\left(p_{i}\right)\right]$ and differentiable on the open interval $\left(w\left(q_{1}\right)\right.$, $\left.w\left(p_{i}\right)\right)$. Then there exists some $\theta_{1}$ in $\left(w\left(q_{1}\right), w\left(p_{i}\right)\right)$ such that:

$$
f^{\prime}\left(\theta_{1}\right)=\frac{f\left(p_{1}\right)-f\left(q_{1}\right)}{w\left(p_{1}\right)-w\left(q_{1}\right)} .
$$

The function $f$ is also continuous on the closed interval $\left[w\left(p_{2}\right), w\left(q_{2}\right)\right]$ and differentiable on the open interval $\left(w\left(p_{2}\right), w\left(q_{2}\right)\right)$. So there also exists some $\theta_{2}$ in $\left(w\left(p_{2}\right)\right.$, $\left.w\left(q_{2}\right)\right)$ such that:

$$
f^{\prime}\left(\theta_{2}\right)=\frac{f\left(q_{2}\right)-f\left(q_{2}\right)}{w\left(p_{2}\right)-w\left(p_{2}\right)} .
$$

We know that $w\left(p_{1}\right)-w\left(q_{1}\right)=w\left(q_{2}\right)-w\left(p_{2}\right)$. We also know that $\theta_{1}<\theta_{2}$ since they are located in different intervals. This means that $f^{\prime}\left(\theta_{1}\right)>f^{\prime}\left(\theta_{2}\right)$ since the function $f$ is concave and the derivative is monotonically decreasing. So $f\left(p_{1}\right)-f\left(q_{1}\right)=$ $f^{\prime}\left(\theta_{1}\right)\left(w\left(p_{1}\right)-w\left(q_{1}\right)\right)=f^{\prime}\left(\theta_{1}\right)\left(w\left(q_{2}\right)-w\left(p_{2}\right)\right)>f^{\prime}\left(\theta_{2}\right)\left(w\left(q_{2}\right)-w\left(p_{2}\right)\right)=f\left(q_{2}\right)-$ $f\left(p_{2}\right)$. Thus $f\left(p_{1}\right)-f\left(q_{1}\right)>f\left(q_{2}\right)-f\left(p_{2}\right)$, and therefore $f\left(p_{1}\right)+f\left(p_{2}\right)>f\left(q_{1}\right)+f\left(q_{2}\right)$. Since $w\left(p_{1}\right)^{r}+w\left(p_{2}\right)^{r}>w\left(q_{1}\right)^{r}+w\left(q_{2}\right)^{r}$, and everything else is being equal, in the situation specified by the condition, $W(A)>W(B)$.

\section{Proofs for the negative social welfare measure}

Let $A$ be a finite population, $p$ a member of $A, n$ the number of individuals in $A, v$ a measure of individual welfare, and $v_{n} \max$ and $v_{n}$ min the highest and lowest possible levels of negative individual welfare, respectively. Then we have:

The negative social welfare measure $W_{N}$

$W_{N}(A, v)=\alpha \frac{-\sum_{i=1}^{n}\left(z\left(p_{i}\right)\right)^{t}}{n^{u}}$, where:

$z\left(p_{i}\right)=\frac{v\left(p_{i}\right)}{v_{n} \max }, t=1+\frac{0.9}{2 y}, u=\frac{1}{2 y}, y=\frac{v_{n} \min }{v_{n} \max }$, where: $\alpha>1, v\left(p_{i}\right) \leq 0, n \geq 2, y$ $\geq 2$.

I will now show that $W_{N}$ satisfies the five conditions. 


\section{Satisfaction of the equivalence condition}

Equivalence Condition: For a measure of social welfare $W$ and for all possible populations $A$ and $B$ and their members $p_{i} \in A$ and $q_{i} \in B$, such that $|A|=B \mid$, if there is a bijection from $A$ to $B$, such that each individual $p_{i} \in A$ could be paired with an individual $q_{i} \in B$ so that for each pair of individuals $\left(p_{i}, q_{i}\right)$ it is the case that $v\left(p_{i}\right)=v\left(q_{i}\right)$, then $A$ does equally well as $B$, and thus $W(A)=W(B)$.

Proof The negative social welfare measure satisfies the Equivalence Condition since it is a symmetric function.

\section{Satisfaction of the dominance condition}

Dominance Condition: For a measure of social welfare $W$ and for all possible populations $A$ and $B$ and their members $p_{i} \in A$ and $q_{i} \in B$, such that $|A|=|B|$, if there is a bijection from $A$ to $B$, such that each individual $p_{i} \in A$ could be paired with an individual $q_{i} \in B$ so that for each pair of individuals $\left(p_{i}, q_{i}\right)$ it is the case that $v\left(p_{i}\right) \geq v\left(q_{i}\right)$, and there is at least one pair of individuals $\left(p_{j}, q_{j}\right)$ for which it is the case that $v\left(p_{j}\right)>v\left(q_{j}\right)$, then $A$ does better than $B$, and thus $W(A)>W(B)$.

Proof It is trivial that the negative social welfare measure satisfies the Dominance Condition, since $\sum_{i=1}^{n} z\left(p_{i}\right)^{t}$ is a strictly increasing function of $z\left(p_{i}\right)$ for every $i$ and $-\sum_{i=1}^{n} z\left(p_{i}\right)^{t}$ therefore is a strictly decreasing function for every $i$ (and $z\left(p_{i}\right)=$ $\left.v\left(p_{i}\right) / v_{n} \max \right)$.

\section{Satisfaction of the quality condition}

Quality Condition: For a measure of social welfare $W$ and for all possible populations $A$ and $B$ and their members $p_{i} \in A$ and $q_{i} \in B$, such that $|A|=$ $n$ and $|B|=m, \Sigma v\left(p_{i}\right)=\Sigma v\left(q_{i}\right)$, and the members of $A$ all have the same level of welfare, and the members of $B$ all have the same level of welfare, if $\Sigma v\left(p_{i}\right) / n$ $>\Sigma v\left(q_{i}\right) / m$, then $A$ does better than $B$, and thus $W(A)>W(B)$.

Proof In this situation, $v\left(p_{i}\right) \leq 0$ and $v\left(q_{i}\right) \leq 0$, and all $v\left(p_{i}\right)$ and all $v\left(q_{i}\right)$ are equal. Since $\Sigma v\left(p_{i}\right) / n>\Sigma v\left(q_{i}\right) / m$, it is the case that $|A|>B \mid$. It is sufficient to prove that the condition holds for $n=m+1$.

Let us use $z\left(p_{i}\right)=v\left(p_{i}\right) / v_{n} \max$ and assume that $z\left(p_{i}\right)=k$, where $1 \leq i \leq m$, and that $z\left(q_{j}\right)=k \frac{m}{m+1}$, where $1 \leq j \leq m+1$. To prove that the negative social welfare measure satisfies the Quality Condition, we need to prove that:

$$
\frac{-\sum_{1}^{n} z\left(p_{i}\right)^{t}}{m^{u}}<\frac{-\sum_{1}^{n} z\left(q_{j}\right)^{t}}{(m+1)^{u}}
$$

which becomes:

$$
\frac{-k^{t} m}{m^{u}}<\frac{-k^{t}\left(\frac{m}{m+1}\right)^{t}(m+1)}{(m+1)^{u}}
$$


which is equivalent to:

$$
m^{1-u-t}>(m+1)^{1-u-t},
$$

which holds since $u+t>1$.

\section{Satisfaction of the strict monotonicity condition}

Strict Monotonicity Condition (negative part): For a measure of social welfare $W$ and for any possible population $A$ and any individual $q \notin A$, if $v(q) \leq-1$, then $A \cup\{q\}$ does worse than $A$ and thus $W(A \cup\{q\})<W(A)$.

Proof The proof is similar to the proof for the positive social welfare measure. To prove that the negative social welfare measure satisfies the Strict Monotonicity Condition, it is sufficient to look at the case where $z(q)=1$. Thus we need to show that, for $t=$ $1+\frac{0.9}{2 y}$, it is the case that:

$$
\frac{-\sum_{i=1}^{n} z\left(p_{i}\right)^{t}}{n^{u}}>\frac{-\left(\sum_{i=1}^{n} z\left(p_{i}\right)^{t}+1^{t}\right)}{(n+1)^{u}} .
$$

This is equivalent to:

$$
1<\frac{n^{u}}{(n+1)^{u}}\left(1+\frac{1}{\sum_{i=1}^{n} z\left(p_{i}\right)^{t}}\right) .
$$

Similarly to the positive case it suffices to prove this for $\left|v\left(p_{i}\right)\right|=\left|v_{n}\right| \max$ and $1 \leq i$ $\leq n$, that is, to prove:

$$
1<\frac{n^{u}}{(n+1)^{u}}\left(1+\frac{1}{n}\left(\frac{\left|v_{n}\right| \min }{\left|v_{n}\right| \max }\right)^{t}\right),
$$

that is, to prove:

$$
1<\left(1+\frac{1}{n}\right)^{-u}\left(1+\frac{1}{n}\left(\frac{1}{y}\right)^{t}\right)
$$

With $u=\frac{1}{2 y}$, it suffices to prove:

$$
1<\left(1+\frac{1}{n}\right)^{-\frac{1}{2 y}}\left(1+\left(\frac{1}{y}\right)^{1+\frac{0.9}{2 y}}\right), \text { for } y \geq 2, n \geq 2 \text {. }
$$

By using $\left(1+\frac{1}{n}\right)^{-\frac{1}{2 y}}>1-\frac{1}{2 n y}$, it suffices to prove:

$$
1<\left(1-\frac{1}{2 n y}\right)\left(1+\frac{1}{n y}\left(\frac{1}{y^{0.9 / 2 y}}\right)\right)
$$


Now, taking the derivative of $y^{\frac{0.9}{2 y}}=e^{(\ln y) \frac{0.9}{2 y}}$ we see that it has a maximum for $y=e$ and $e^{\frac{0.9}{2 e}}<1.3$. So for $n \geq 2, y \geq 2:\left(1-\frac{1}{2 n y}\right)\left(1+\frac{1}{n y}\left(\frac{1}{y^{0.9 / 2 y}}\right)\right)>$ $\left(1-\frac{1}{2 n y}\right)\left(1+\frac{1}{1.3 n y}\right)=1+\left(\frac{1}{1.3}-\frac{1}{2}\right)\left(\frac{1}{n y}\right)-\frac{1}{2.6 n^{2} y^{2}}=1+\left(\frac{1}{1.3}-\frac{1}{2}-\frac{1}{2.6 n y}\right)$ $\left(\frac{1}{n y}\right)>1+\left(\frac{1}{1.3}-\frac{1}{2}-\frac{1}{10}\right)\left(\frac{1}{n y}\right)>1+0.15 \frac{1}{n y}>1$. So (14) is proved and the Strict Monotonicity Condition holds.

\section{Satisfaction of the limited equality condition}

Limited Equality Condition: For a measure of social welfare $W$ and for all possible populations $A$ and $B$ and their members $p_{i} \in A$ and $q_{i} \in B$, such that $|A|=|B|$ and $\Sigma v\left(p_{i}\right)=\Sigma v\left(q_{i}\right)$, if there is a bijection from $A$ to $B$, such that each individual $p_{i} \in A$ could be paired with an individual $q_{i} \in B$ so that for each pair of individuals $\left(p_{i}, q_{i}\right)$ it is the case that $v\left(p_{i}\right)=v\left(q_{i}\right)$, except for four individuals: $p_{1}, p_{2}, q_{1}, q_{2}$, such that $\left|v\left(p_{1}\right)-v\left(p_{2}\right)\right|<\left|v\left(q_{1}\right)-v\left(q_{2}\right)\right|$, then $A$ does better than $B$, and thus $W(A)>W(B)$.

Proof That the negative social welfare measure satisfies the Limited Equality Condition can be shown by using the same proof that was used for the positive social welfare measure, since a negative function of a strictly convex function is a strictly concave function.

\section{Proofs for the overall social welfare measure}

Let $W_{P}$ be a measure of positive social welfare, $W_{N}$ a measure of negative social welfare, $A$ a finite population, $p$ a member of $A, n$ the number of individuals in $A$, $v$ a measure of individual welfare, $v_{p} \max$ and $v_{n}$ min the highest and lowest possible levels of individual welfare, $v_{p}$ min the lowest possible level of individual welfare that is still positive, and $v_{n} \max$ the highest possible level of individual welfare that is still negative, $x$ the ratio between $v_{p} \max$ and $v_{p} \min$, and $y$ the ratio between $v_{n} \min$ and $v_{n} \max$. Then we have:

The overall social welfare measure $W$

$W(A, v)=W_{P}(A, v)+W_{N}(A, v)$, where:

$W_{P}(A, v)=\frac{\sum_{i=1}^{n}\left(w\left(p_{i}\right)\right)^{r}}{n^{s}}$, where:

$w\left(p_{i}\right)=\frac{v\left(p_{i}\right)}{v_{p} \min }, r=1-\frac{0.9}{2 x}, s=\frac{1}{2 x}, x=\frac{v_{p} \max }{v_{p} \min }$, and where: $v\left(p_{i}\right) \geq 0, n \geq 2, x$

$\geq 2$,

and

$W_{N}(A, v)=\alpha \frac{-\sum_{i=1}^{n}\left(z\left(p_{i}\right)\right)^{t}}{n^{u}}$, where:

$z\left(p_{i}\right)=\frac{v\left(p_{i}\right)}{v_{n} \max }, t=1+\frac{0.9}{2 y}, u=\frac{1}{2 y}, y=\frac{v_{n} \min }{v_{n} \max }$, where: $\alpha>1, v\left(p_{i}\right) \leq 0, n \geq 2, y \geq 2$, $v_{p} \min =\left|v_{n} \max \right|$, and $v_{p} \max =\left|v_{n} \min \right|$.

I will last show that $W$ satisfies the six conditions. 


\section{Satisfaction of the equivalence condition}

Equivalence Condition: For a measure of social welfare $W$ and for all possible populations $A$ and $B$ and their members $p_{i} \in A$ and $q_{i} \in B$, such that $|A|=|B|$, if there is a bijection from $A$ to $B$, such that each individual $p_{i} \in A$ could be paired with an individual $q_{i} \in B$ so that for each pair of individuals $\left(p_{i}, q_{i}\right)$ it is the case that $v\left(p_{i}\right)=v\left(q_{i}\right)$, then $A$ does equally well as $B$, and thus $W(A)=W(B)$.

Proof The overall social welfare measure satisfies the Equivalence Condition since it is a symmetric function.

\section{Satisfaction of the dominance condition}

Dominance Condition: For a measure of social welfare $W$ and for all possible populations $A$ and $B$ and their members $p_{i} \in A$ and $q_{i} \in B$, such that $|A|=|B|$, if there is a bijection from $A$ to $B$, such that each individual $p_{i} \in A$ could be paired with an individual $q_{i} \in B$ so that for each pair of individuals $\left(p_{i}, q_{i}\right)$ it is the case that $v\left(p_{i}\right) \geq v\left(q_{i}\right)$, and there is at least one pair of individuals $\left(p_{j}, q_{j}\right)$ for which it is the case that $v\left(p_{j}\right)>v\left(q_{j}\right)$, then $A$ does better than $B$, and thus $W(A)>W(B)$.

Proof It is trivial that the overall social welfare measure satisfies the Dominance Condition, since it is composed of two strictly increasing functions.

\section{Satisfaction of the quality condition}

Quality Condition: For a measure of social welfare $W$ and for all possible populations $A$ and $B$ and their members $p_{i} \in A$ and $q_{i} \in B$, such that $|A|=$ $n$ and $|B|=m, \Sigma v\left(p_{i}\right)=\Sigma v\left(q_{i}\right)$, and the members of $A$ all have the same level of welfare, and the members of $B$ all have the same level of welfare, if $\Sigma v\left(p_{i}\right) / n$ $>\Sigma v\left(q_{i}\right) / m$, then $A$ does better than $B$, and thus $W(A)>W(B)$.

Proof: Let us use normalized values instead of the values of $v$. We use $w$ for both positive and negative values (rather than $w$ for positive and $z$ for negative). There are three relevant cases:

i) $w\left(p_{i}\right) \geq 0$ and $w\left(q_{i}\right) \geq 0$,

ii) $w\left(p_{i}\right) \leq 0$ and $w\left(q_{i}\right) \leq 0$,

iii) $w\left(p_{i}\right) \geq 0$ and $w\left(q_{i}\right) \leq 0$.

In the first case the proposed measure satisfies the Quality Condition since the positive part of the measure satisfies the condition. In the second case it satisfies the condition since the negative part of the measure satisfies the condition. In the third case it trivially satisfies the condition since the measure gives the result that $W(A)>0$ and $W(B)<0$. 


\section{Satisfaction of the strict monotonicity condition}

Strict Monotonicity Condition: For a measure of social welfare $W$ and for any possible population $A$ and any individual $q \notin A$, if $v(q) \geq 1$ then $A \cup\{q\}$ does better than $A$ and thus $W(A \cup\{q\})>W(A)$ and if $v(q) \leq-1$, then $A \cup\{q\}$ does worse than $A$ and thus $W(A \cup\{q\})<W(A)$.

Proof That the overall social welfare measure satisfies the Strict Monotonicity Condition follows from the fact that both the positive and negative parts of the measure satisfy the condition.

\section{Satisfaction of the limited equality condition}

Limited Equality Condition: For a measure of social welfare $W$ and for all possible populations $A$ and $B$ and their members $p_{i} \in A$ and $q_{i} \in B$, such that $|A|=|B|$ and $\Sigma v\left(p_{i}\right)=\Sigma v\left(q_{i}\right)$, if there is a bijection from $A$ to $B$, such that each individual $p_{i} \in A$ could be paired with an individual $q_{i} \in B$ so that for each pair of individuals $\left(p_{i}, q_{i}\right)$ it is the case that $v\left(p_{i}\right)=v\left(q_{i}\right)$, except for four individuals: $p_{1}, p_{2}, q_{1}, q_{2}$, such that $\left|v\left(p_{1}\right)-v\left(p_{2}\right)\right|<\left|v\left(q_{1}\right)-v\left(q_{2}\right)\right|$, then $A$ does better than $B$, and thus $W(A)>W(B)$.

Proof We may prove that the measure satisfies the Limited Equality Condition in two steps: first we show that the Limited Equality Condition follows from a Priority Condition. Next we show that the measure satisfies the Priority Condition (and consequently also the Limited Equality Condition). This is the condition in question:

Priority Condition: For a measure of social welfare $W$ and for any possible population $C$ and for any individuals $r_{i}, s_{i} \in C$ such that $v\left(r_{i}\right)<v\left(s_{i}\right)$, if it is possible to either raise the welfare of $r_{i}$ by $m$, resulting in population $C^{*}$ or raise the welfare of $s_{i}$ by $m$, resulting in population $C^{* *}$, then $C^{*}$ would fare better than $C^{* *}$ and thus $W\left(C^{*}\right)>W\left(C^{* *}\right)$.

To show that the Limited Equality Condition follows from the Priority Condition, we first assume that $v\left(q_{1}\right)<v\left(p_{1}\right) \leq v\left(p_{2}\right)<v\left(q_{2}\right)$ (which we can do without loss of generality).

Next, consider a population $C$, where $|C|=|A|=|B|$, and members $r_{1}$ and $s_{1}$, such that $v\left(r_{1}\right)=v\left(q_{1}\right)$, and $v\left(s_{1}\right)=v\left(p_{2}\right)$, and for all other $i, v\left(r_{i}\right)=v\left(s_{i}\right)=v\left(p_{i}\right)=v\left(q_{i}\right)$. Since $v\left(q_{1}\right)+v\left(q_{2}\right)=v\left(p_{1}\right)+v\left(p_{2}\right)$, we get $v\left(q_{2}\right)-v\left(p_{2}\right)=v\left(p_{1}\right)-v\left(q_{1}\right)$ which gives:

$$
v\left(q_{2}\right)-v\left(s_{1}\right)=v\left(p_{1}\right)-v\left(r_{1}\right)
$$

We see that $A$ is gotten from $C$ by increasing $v\left(r_{1}\right)$ to

$$
v\left(p_{1}\right)=v\left(r_{1}\right)+\left(w\left(p_{1}\right)-w\left(r_{1}\right)\right)
$$


We also see that $B$ is gotten from $C$ by increasing $v\left(s_{1}\right)=v\left(p_{2}\right)$ to

$$
v\left(q_{2}\right)=v\left(s_{1}\right)+\left(w\left(q_{2}\right)-w\left(s_{1}\right)\right)
$$

Since $v\left(r_{1}\right)<v\left(s_{1}\right)$ and $\left(w\left(p_{1}\right)-w\left(r_{1}\right)\right)=\left(w\left(q_{2}\right)-w\left(s_{1}\right)\right)$ and (16) and (17) holds, it is the case that if $W$ satisfies the Priority Condition, then $W(A)>W(B)$.

Thus the Limited Equality Condition has been shown to follow from the Priority Condition, and we next show that the measure satisfies the Priority Condition. To show this we must prove that if $m>0$ and $v(p)<v(q)$, then $W\left(A^{*}\right)>W\left(A^{* *}\right)$, where $A *$ is the possible population where the original value $v(p)$ is replaced by $v(p)+m$, and $A^{* *}$ is the possible population where the original value $v(q)$ is replaced by $v(q)+$ $m$.

Let us again use normalized values instead of the values of $v$ and use $w$ for both positive and negative values. Since $W(A)=W_{P}(A)+W_{N}(A), W\left(A^{*}\right)=W_{P}\left(A^{*}\right)+$ $W_{N}\left(A^{*}\right)$, and $W\left(A^{* *}\right)=W_{P}\left(A^{* *}\right)+W_{N}\left(A^{* *}\right)$, we must consider the cases (i) to (vi) below:

i) $w(p) \geq 0$ and $w(q) \geq 1$ and $w(p)+m \geq 1$ and $v(q)+m>1$,

ii) $w(p)<-1$ and $w(q) \leq-1$ and $w(p)+m<0$ and $w(q)+m \leq 0$,

iii) $w(p)<-1$ and $w(q) \leq-1$ and $w(p)+m \leq 0$ and $w(q)+m \geq 1$,

iv) $w(p)<-1$ and $w(q) \leq-1$ and $w(p)+m \geq 1$ and $w(q)+m>1$,

v) $w(p) \leq-1$ and $w(q) \geq 0$ and $w(p)+m \leq 0$ and $w(q)+m \geq 1$,

vi) $w(p) \leq-1$ and $w(q) \geq 0$ and $w(p)+m \geq 1$ and $w(q)+m>1$.

First case (i): the case where $w(p) \geq 0$, and it must be the case that $w(q) \geq 1$, since $w(p)<w(q)$ and 1 is the smallest possible positive value of $w(r)$ for any $r$. In this case $W_{N}(A)=W_{N}\left(A^{*}\right)=W_{N}\left(A^{* *}\right)$, so we only need to consider the changes of $W_{P}(A)$ when $A$ is replaced by $A^{*}$ and $A^{* *}$, respectively. We have:

$$
\begin{aligned}
W_{p}\left(A^{*}\right)-W_{p}(A)= & \frac{(w(p)+m)^{r}-(w(p))^{r}}{n^{s}}>\frac{(w(q)+m)^{r}-(w(q))^{r}}{n^{s}} \\
& =W_{p}\left(A^{* *}\right)-W_{p}(A) .
\end{aligned}
$$

Thus $W_{P}\left(A^{*}\right)>W_{P}\left(A^{* *}\right)$. Therefore $W\left(A^{*}\right)=W_{P}\left(A^{*}\right)+W_{N}\left(A^{*}\right)=W_{P}\left(A^{*}\right)+$ $W_{N}(A)>W_{P}\left(A^{* *}\right)+W_{N}(A)=W_{P}\left(A^{* *}\right)+W_{N}\left(A^{* *}\right)=W_{P}\left(A^{* *}\right)$. Thus $W\left(A^{*}\right)>$ $W(A * *)$.

Second case (ii): the case where $w(p)+m<w(q)+m \leq 0$ and thus $w(p)<w(q)$ $<0$. Obviously, in this case: $W_{P}(A)=W_{P}\left(A^{*}\right)=W_{P}\left(A^{* *}\right)$, so we only need to look at the negative part of the overall social welfare measure. That $W\left(A^{*}\right)>W\left(A^{* *}\right)$ may then be proved in an analogous way to the previous case.

Next we have to consider the cases where the changes from $A$ to either $A^{*}$ or $A^{* *}$ may affect both the positive and negative parts of the overall social welfare measure. We shall first consider the third case.

Third case (iii): the case where $w(p) \leq-1$ and $w(p)+m \leq 0, w(q) \leq-1$ and $w(q)$ $+m>0$. In this case we get:

$W\left(A^{*}\right)-W(A)=\frac{|w(p)|-|w(p)+m|^{t}}{n^{u}}$ and $W\left(A^{* *}\right)-W(A)=\frac{|w(q)+m|^{t}}{n^{s}}+\frac{|w(q)|^{t}}{n^{u}}$

This gives: 
$W\left(A^{*}\right)-W\left(A^{* *}\right)=\frac{|w(p)|^{t}-|w(p)+m|^{t}}{n^{u}}-\frac{|w(q)+m|^{t}}{n^{s}}-\frac{|w(q)|^{t}}{n^{u}}>\frac{|w(p)|^{t}-|w(p)+m|^{t}}{n^{u}}-$ $\frac{|w(q)+m|^{t}}{n^{u}}-\frac{|w(q)|^{t}}{n^{u}}=C$,

since $r<1<t$ and $u=s$. Now, since $w(p), w(p)+m$ and $w(q)$ are all negative, we get, if we put $|w(p)+m|=a$ and $|w(q)|=b$, that $b<m$ and $|w(p)|=a+m$ and $\mid w(q)$ $+m l=m-b$. So with this we get:

$$
W\left(A^{*}\right)-W\left(A^{* *}\right)>C=\frac{1}{n^{u}}\left((a+m)^{t}-a^{t}-(m-b)^{t}-b^{t}\right) .
$$

Now, since $t>1$, we get:

$$
(a+m)^{t}=(a+(m-b)+b)^{t}>a^{t}+(m-b)^{t}+b^{t} \text { and so } W\left(A^{*}\right)-W\left(A^{* *}\right)>0,
$$
and thus $W\left(A^{*}\right)>W\left(A^{* *}\right)$.

Fourth case (iv): the case where $w(p) \leq-1$ and $w(p)+m \geq 1, w(q) \leq-1$ and $w(q)+m \geq 1$. This gives:

$$
W\left(A^{*}\right)-W(A)=\frac{1}{n^{s}}(w(p)+m)^{r}+\frac{1}{n^{u}}(|w(p)|)^{t} .
$$

and

$$
W\left(A^{* *}\right)-W(A)=\frac{1}{n^{s}}(w(q)+m)^{r}+\frac{1}{n^{u}}(|w(q)|)^{t}
$$

Thus

$$
W\left(A^{*}\right)-W\left(A^{* *}\right)=\frac{1}{n^{s}}\left[(w(p)+m)^{r}-(w(q)+m)^{r}\right]+\frac{1}{n^{u}}\left[|w(p)|^{t}-|w(q)|^{t}\right],
$$

where $|w(p)|>|w(q)|$. Since $r<1<t, u=s, w(p)+m \geq 1$ and $w(q)+m \geq 1$, we have: $\left|\frac{1}{n^{s}}(w(p)+m)^{r}-(w(q)+m)^{r}\right| \leq \frac{1}{n^{s}}|(w(p)+m)-(w(q)+m)|=\frac{1}{n^{s}}|w(p)-w(q)|=$ $\frac{1}{n^{u}}|w(p)-w(q)|=\frac{1}{n^{u}}(|w(p)|-|w(q)|)<\frac{1}{n^{u}}\left(|w(p)|^{t}-|w(q)|^{t}\right)$.

Thus $W\left(A^{*}\right)>W\left(A^{* *}\right)$.

Fifth case (v): the case where $w(p) \leq-1$ and $w(p)+m \leq 0, w(q) \geq 0$ and $w(q)+m>$ 0 . Here $W\left(A^{*}\right)-W(A)=\frac{1}{n^{u}}\left(|w(p)|^{t}-|w(p)+m|^{t}\right)>\frac{1}{n^{u}}(|w(p)|-|w(p)+m|)=$ $\frac{m}{n^{u}}=\frac{m}{n^{s}}>\frac{(w(q)+m)^{r}-w(q)^{r}}{n^{s}}=W\left(A^{* *}\right)-W(A)$, so $W\left(A^{*}\right)>W\left(A^{*}\right)$.

Sixth and final case: the case where $w(p) \leq-1$ and $w(p)+m \geq 1, w(q) \geq 0$ and $w(q)$ $+m>0$. This gives:

$$
W\left(A^{*}\right)-W(A)=\frac{1}{n^{u}}|w(p)|^{t}+\frac{(w(p)+m)^{r}}{n^{s}}
$$

and

$$
W\left(A^{* *}\right)-W(A)=\frac{(w(p)+m)^{r}}{n^{s}}-\frac{w(q)^{r}}{n^{s}}<\frac{m^{r}}{n^{s}} .
$$


It is the case that:

$$
\begin{aligned}
W\left(A^{*}\right)-W(A) & =\frac{1}{n^{u}}|w(p)|^{t}+\frac{(w(p)+m)^{r}}{n^{s}}=\frac{1}{n^{u}}|w(p)|^{t}+\frac{(m-|w(p)|)^{r}}{n^{s}} \\
& =\frac{1}{n^{s}}|w(p)|^{t}+\frac{(m-|w(p)|)^{r}}{n^{s}}>\frac{m^{r}}{n^{s}}-W(A) .
\end{aligned}
$$

Thus $W\left(A^{*}\right)>W\left(A^{* *}\right)$ also in the last case.

\section{Satisfaction of the asymmetry condition}

Asymmetry condition: For a measure of social welfare $W$ and for all possible populations $A$ and $B$ if there is a bijection from $A$ to $B$, such that each individual $p_{i}$ $\in A$ could be paired with an individual $q_{i} \in B$ so that for each pair of individuals $\left(p_{i}, q_{i}\right)$ it is the case that $\left|v\left(p_{i}\right)\right|=\left|v\left(q_{i}\right)\right|$ and also the case that $v\left(p_{i}\right)>v\left(q_{i}\right)$, then $|W(A)|<|W(B)|$.

Proof It is trivial that the measure satisfies the Asymmetry Condition. It does so because the negative social welfare measure is multiplied by a factor $\alpha>1$, and because individual welfare values are aggregated by a concave function and individual illfare values are aggregated by a convex function.

\section{References}

Acton HB, Watkins JWN (1963) Symposium: negative utilitarianism. Proc Aristotelian Soc Suppl 37:83-114

Anglin B (1977) The repugnant conclusion. Can J Philos 7(4):745-754

Arrhenius G (2000) Future generations: a challenge for moral theory. Dissertation. Uppsala University, Uppsala

Bentham J (1823) An introduction to the principles of morals and legislation, 2nd edn. Pickering, London Blackorby C, Donaldson D (1984) Social criteria for evaluating population change. J Publ Econ 25(1-2):13-33

Blackorby C, Bossert W, Donaldson D, Fleurbauey M (1998) Critical levels and the (reverse) repugnant conclusion. J Econ 67(1):1-15

Broad CD (1930) Five types of ethical theory. Kegan Paul, Trench, Trubner \& Co, London Broome J (1991) Weighing goods: equality, uncertainty and time. Basil Blackwell, Oxford Broome J (1999) Ethics out of economics. Cambridge University Press, Cambridge

Broome J (2004) Weighing lives. Oxford University Press, Oxford

Broome J (2015) Equality versus priority: a useful distinction. Econ Philosophy 31(2):219-228

Brown C (2007) Prioritarianism for variable populations. Philos Stud 134(3):325-361

Brülde B (2007) Happiness and the good life: introduction and conceptual framework. J Happiness Stud $8(1): 1-14$

Carlson E (1998) Mere addition and two trilemmas of population ethics. Econ Philosophy 14(2):283-306

Christiano T, Braynen W (2008) Inequality, injustice and levelling down. Ratio. New Series. 21(4):393-420

Cowen T (1996) What do we learn from the repugnant conclusion? Ethics 106(4):754-775

Cowen T (2004) Resolving the repugnant conclusion. In: Ryberg J, Tännsjö T (eds) The repugnant conclusion. Kluwer, Dordrecht, pp 81-97

Dalton H (1920) The measurement of the inequality of incomes. Econ J 30(119):348-361

Dasgupta P (1988) Lives and well-being. Soc Choice Welfare 5(2-3):103-126

Edgeworth FY (1877) New and old methods of ethics: or physical ethics and methods of ethics. James Parker \& Co, Oxford 
Gauthier D (1963) Practical reasoning. Clarendon Press, Oxford

Gini C (1912) Variabilità e mutabilità. In: Studi economico-giuridici della Facoltà di Giurisprudenza. Università di Cagliari, Bologna

Griffin J (1979) Is unhappiness morally more important than happiness? Philosophical Q 29(114):47-55

Griffin J (1986) Well-being: its meaning, measurement, and moral importance. Clarendon Press, Oxford

Hammond PJ (1976) Equity, Arrow's conditions and Rawls difference principle. Econometrica 44(4):793-804

Hare R (1993) Possible people. In: Hare R (ed) Essays on bioethics. Clarendon Press, Oxford, pp 67-83

Harsanyi JC (1955) Cardinal welfare, individualistic ethics and interpersonal comparisons of utility. J Political Econ 63(4):309-321

Hirose I (2009) Reconsidering the value of equality. Aust J Philosophy 87(2):301-312

Holtug N (2004) Person-affecting moralities. In: Ryberg J, Tännsjö T (eds) The repugnant conclusion. Kluwer, Dordrecht, pp 129-161

Holtug N (2007) Prioritarianism. In: Holtug N, Lippert-Rasmussen K (eds) Egalitarianism: new essays on the nature and value of equality. Clarendon Press, Oxford, pp 125-156

Holtug N (2010) Persons, interests and justice. Oxford University Press, Oxford

Huemer M (2008) In defence of repugnance. Mind 117(468):899-933

Hutcheson F (1738) An inquiry into the original of our ideas of beauty and virtue, in two treatises, 4th edn. D. Midwinter, A. Bettesworth, C. Hitch, J. and J. Pemberton, R. Ware, C. Rivington, F. Clay, A. Ward, J. and P. Knapto, T. Longman, R. Hett, and J. Wood. London

von Leibniz G (1768) Observationes de principio juris. In: Gothofredi Guillelmi Leibnitii Opera Omnia, Geneve

Locke D (1987) The Parfit population problem. Philosophy 62(240):131-157

Mackie JL (1985) The Parfit population problem. In: Mackie JL (ed) Persons and values, vol 2. Clarendon Press, Oxford, pp 242-248

McMahan J (1981) Problems of population theory. Ethics 92(1):96-127

McTaggart J (1927) The nature of existence, vol 2. Cambridge University Press, Cambridge

Mill JS (1867) Utilitarianism, 3d edn. Longmans, Green, Reader and Dyer

Mulgan T (2002) The reverse repugnant conclusion. Utilitas 14(3):360-364

Mulgan T (2006) Future people. Oxford University Press, Oxford

Nagel T (1991) Equality and partiality. Oxford University Press, Oxford

Narveson J (1967) Morality and utility. John Hopkins Press, Baltimore

$\mathrm{Ng}$ Y-K (1989) What should we do about future generations? Impossibility of Parfit's theory X. Econ Philosophy 5(2):235-253

Österberg J (2006) Value and existence: the problem of future generations. In: Lindström S, Sliwinski R, Österberg J (eds) Odds and ends. Department of Philosophy, Uppsala University, Uppsala, pp 94-10

Pareto V (1896) Cours d'économie politique professé à l'université de Lausanne. F. Rouge, Lausanne Pareto V (1909) Manuel d'economie politique. Giard et. E, Paris

Parfit D (1982) Future generations; further problems. Philos Public Aff 11(2):113-172

Parfit D (1984) Reasons and persons. Oxford University Press, Oxford

Parfit D (1986) Overpopulation and the quality of life. In: Singer P (ed) Applied ethics. Oxford University Press, Oxford, pp 145-164

Persson I (2004) The root of the repugnant conclusion and its rebuttal. In: Ryberg J, Tännsjö T (eds) The repugnant conclusion. Kluwer, Dordrecht, pp 187-199

Pietra G (1915) Delle relazioni tra gli indici di variabilitià, note I e II. Atti del Reale Istituto Veneto di Scienze, Lettere ed Arti 74:775-804

Pigou AC (1912) Wealth and welfare. Macmillan, London

Popper K (1945) The open society and its enemies. Routledge, London

Pressman M (2015) A defence of average utilitarianism. Utilitas 27(4):389-424

Rabinowicz W (2009) Broome and the intuition of neutrality. Philosophical Issues 19(1):389-411

Rachels S (1998) Is it good to make happy people? Bioethics 12(2):93-110

Rachels S (2001) A set of solutions to Parfit's problems. Nôus 35(2):214-238

Rawls J (1971) A theory of justice. Harvard University Press, Cambridge, MA

Ryberg J (1996) Is the repugnant conclusion repugnant? Philosophical Papers 25(3):161-177

Sen A (1970) Collective choice and social welfare. Harvard University Press, Cambridge, MA

Sen A (1973) On economic inequality. Clarendon Press, Oxford 
Sen A (1977) On weights and measures: informational constraints in social welfare analysis. Econometrica 45(7):1539-1572

Sidgwick H (1874) The methods of ethics. Macmillian, London

Sikora RI (1975) Utilitarianism: the classical principle and the average principle. Can J Philos 5(3):409-419

Sikora RI (1978) Is it wrong to prevent the existence of future generations?. In: Barry B, Sikora RI (eds) Obligations to future generations, Temple University Press, Philadelphia, pp 112-166

Smart JJC (1961) Outline of a system of utilitarian ethics. Cambridge University Press, Melbourne

Smart RN (1958) Negative utilitarianism. Mind 67(268):542-543

Sumner LW (1978) Classical utilitarianism and the population optimum. In: Barry B and Sikora RI (eds) Obligations to future generations, pp 91-111

Temkin L (1993) Inequality. Oxford University Press, Oxford

Theil H (1967) Economics and information theory. North Holland, Amsterdam

Tungodden B (2003) The value of equality. Econ Philosophy 19(1):1-44

Tännsjö T (2002) Why we ought to accept the repugnant conclusion. Utilitas 12(3):339-359

Walker ADM (1974) Negative utilitarianism. Mind New Series 83(331):424-428

Weirich P (1983) Utility tempered with equality. Nous 17(3):423-439

Wicksell K (1973) The optimum of population (1910). In: Overbeek J (ed) Wiksell on population. Econ Dev Cult Change 21(2):205-211

Publisher's Note Springer Nature remains neutral with regard to jurisdictional claims in published maps and institutional affiliations. 